\title{
SALICYLIC ACID EFFECT ON CADMIUM-INDUCED ACCUMULATION OF MINERAL CONTENT IN LEAVES OF PISTACHIO SPECIES FROM TURKEY: AN ANALYSIS COUPLED WITH CHEMOMETRICS AND MULTIPLE REGRESSION ANALYSIS
}

\author{
Cetinkaya, . $^{{ }^{*}}-$ KUlaK, $M .^{2,3}$ \\ ${ }^{I}$ Department of Horticulture, Faculty of Agriculture, Kilis 7 Aralik University, Kilis, Turkey \\ ${ }^{2}$ Department of Nursing, Yusuf Serefoglu Faculty of Health, Kilis 7 Aralik University, Kilis, \\ Turkey \\ ${ }^{3}$ Advanced Technology Application and Research Center, Kilis 7 Aralik University, Kilis, \\ Turkey \\ ${ }^{*}$ Corresponding author \\ e-mail: hcetinkaya67@gmail.com; phone:+90-348-814-2666/7214 \\ (Received 24 $4^{\text {th }}$ Feb 2019; accepted $10^{\text {th }}$ Apr 2019)
}

\begin{abstract}
Cadmium (Cd) is highly toxic and causes detrimental effects on plants but the adverse effects of $\mathrm{Cd}$ can be alleviated by exogenous applications. Hence, in the current study, the possible alleviative roles of salicylic acid (SA) on accumulation of $\mathrm{Cd}$ were investigated in leaves of pistachio species. Mineral accumulation variations in leaves were tested by analysis of variance (ANOVA) followed by multiple comparison test of Duncan using SPSS and chemometric analysis. For the present study, two-year old pistachio species (Pistacia vera, Pistacia khinjuk, Pistacia terebinthus) were exposed to the combination $\mathrm{Cd}(50$ and $100 \mu \mathrm{M})$ from root and SA $(0.5 \mu \mathrm{M})$ with foliar applications. Accordingly, both SA treatment and Cd had significant effects on accumulation of minerals but SA acid was found to have a little alleviative effect on accumulation $\mathrm{Cd}$. Of the investigated elements, $\mathrm{P}(2.151$ fold-change; $\mathrm{p}<0.001)$ and $\mathrm{Cu}(4.702$ fold-change; $\mathrm{p}<0.001)$ were significant and highly variable over-accumulated elements. According to the principal component analysis (PCA), the multivariate data processing for elements allowing for a large group of diverse data samples was explained in $23.95 \%$ and $22.61 \%$ as the first and second principal components $\left(\mathrm{PC}_{1}\right.$ and $\left.\mathrm{PC}_{2}\right)$. Two components extracted were describing approximately $46.55 \%$ of the common variance.
\end{abstract}

Keywords: macro and micro element, cadmium stress, foliar salicylic acid application, environmental health, Pistacia vera, Pistacia khinjuk, Pistacia terebinthus

\section{Introduction}

Expansion of agricultural areas, increment of soil pollutants, chemical fertilizers, uses of wastewater in agriculture and industrialization have led to pollution of agricultural lands. Although heavy metals are not essential elements for plants, they are transported from soil to the plant and subsequently pose a threat to the health of other livings through food chain. Of those heavy metals, $\mathrm{Cd}$ as a non-essential element to plants is a highly toxic and persistent environmental poison for plants and animals (di Toppi and Gabbrielli, 1999).

Cd accumulation causes structural, physiological and biochemical changes in plants (Khan et al., 2007; Feng et al., 2010). Disruption of the water balance of the plant by affecting stomatal movements, degradation of carbohydrate and interruption of photosynthesis mechanisms are of the consequences after $\mathrm{Cd}$ uptake. Accordingly, 
decrease in yield has been deemed to be associated with $\mathrm{Cd}$ accumulation (Mobin et al., 2007; Hossain et al., 2010; Shie et al., 2010). Cd hinders many cellular functions and inhibits essential activities through complex formation with proteins. Albeit those cytoplasmic toxicity mechanisms are similar in all organisms, different plant species and their varieties, populations and genotypes exhibit a wide range of plasticity in response to the $\mathrm{Cd}$ accumulation (McGrath et al., 2001). We should note that even sensitive or tolerant species also vary considerably for their responses against $\mathrm{Cd}$.

As a response to unfavorable conditions, signal molecules are synthesized and subsequently activate a range of signal transduction pathways in complex plant system. Furthermore, the plausible protective roles of some signal molecules are known in helping plant to cope with the stress (Ganesan and Thomas, 2001). Calcium, jasmonic, ethylene and ethylene and salicylic acid are of the identified signaling molecules (El Tayeb and Ahmed, 2010). Of the identified molecules, the protective roles of salicylic acid (SA) as a defense signal transducer or messenger under stress conditions has been well-reported (Raskin, 1992; Klessig and Malamy, 1994; Ganesan and Thomas, 2001). SA is a hormone-like and phenolic structured molecule modulating and regulating biochemical, physiological and molecular processes of the plants and then favors plant growth and development. It is worthy to note that SA should not be narrowly discussed as an endogenous molecule but also defined, described and discussed as a potent molecule vital to plant systems once used an exogenous regulator under optimal conditions including optimal concentration, mode of applications, appropriate application time etc. for plants (Kulak, 2018). In many studies with different agricultural crops, the role of exogenous SA application has been well-documented in regulation of physiological processes in plants, such as stomatal closure, ion uptake and transport, membrane permeability, and photosynthesis and growth under stress conditions (Shakirova and Bezrukova, 1997; Mishra and Choudhuri, 1999; Pa'1 et al., 2002; Sakhabutdinova et al., 2003; Shakirova et al., 2003; Bhupinder and Usha, 2003). However, there are a few studies on the salicylic acid and cadmium interaction for fruit trees.

For the horticultural plant and crops, rootstocks are of the essential and fundamental issues. In this context, they have been screened and used for propagating temperate fruit trees more than 2000 years due to their influence on scion vigor, cropping, fruit quality, climatic adaptability, and susceptibility to pests and diseases (Webster, 1995; Acar et al., 2017a). In the studies reported by (Acar et al., 2017a, 2017b), the distribution and rootstocks of pistachio have been well-documented, informing that Pistacia genus (Anacardiaceae family) consists of at least eleven species. Of those species, P. mexicana and $P$. texana are of USA and Mexico originated. The other species are mainly distributed within the Mediterranean region, Western and Central Asia and the Middle East (EsmailPour, 2001). Turkey is of the important pistachio producer and suppliers in the world and possesses many wild species of pistachio nut. Of those species, $P$. vera, P. terebinthus, $P$. khinjuk, $P$. atlantica, P. mutica, P. palaestina and P. lentiscus exhibit distribution in different regions of Turkey. The main pistachio rootstock used in Turkey is $P$. vera, and followed by $P$. khinjuk, P. terebinthus and P. atlantica (Acar et al., 2017b).

Of the environmental factors, heavy metals are well-documented to possess potentially deleterious effects on fruit quality of the plants and subsequently on human health. The response of the grafted plants against stress conditions owing to the nutrient status and the presence of heavy metals in the root environment may differ than that of self-rooted plants and may be dependent on the rootstock genotype (Savvas et al., 2010, 2011). As 
highlighted by Savvas et al. (2011), appropriate rootstocks selection may be significant step for restriction of heavy metal accumulation in the aerial parts of the plants.

In this context, we monitored the nutritional status of three rootstocks namely, $P$. vera, $P$. terebinthus and $P$. khinjuk in response to cadmium treatments by addition of foliar salicylic treatments. Along with the study, the results were analyzed using chemometrics and multiple regression analysis in order to discriminate and identify the differences between pistachio rootstocks.

\section{Material and Methods}

\section{Plant material, growth conditions, salicylic acid and Cd application}

In the study, 2-year old seedlings of Pistacia vera, Pistacia terebinthus and Pistaciakhinjuk pistachio species were used. The experiment was carried out in greenhouses of Kilis 7 Aralık University Agricultural Application and Research Center (Turkey). Experiments were conducted in a greenhouse with a $14 \mathrm{~h}$ photoperiod. Mean temperature and relative humidity were $26-30^{\circ} \mathrm{C}$ during day and $16-20^{\circ} \mathrm{C}$ at night, $60 \%$ respectively. The experiments were performed with the replicates including ten pistachio seedlings for each replicate. The physical and chemical properties of experimental soil were as follows: $\mathrm{pH}(8.15$; alkali); water saturation (clay loam); calcium carbonate $\left(\mathrm{CaCO}_{3}\right)$ content $(47.49 \%$; very calcareous); total salt $(0.016 \%$; low salt $)$, phosphorus $\left(\mathrm{P}_{2} \mathrm{O}_{5}\right)(4.12 \mathrm{~kg} / \mathrm{ha}$; low); organic matter $(2.11 \%$; medium $)$. For the experimental group, pistachio seedlings were regularly root-irrigated with two different concentration of cadmium alone $\left(\mathrm{Cd}_{1}: 50 \mu \mathrm{M} ; \mathrm{Cd}_{2}: 100 \mu \mathrm{M}\right)$ for three weeks. Furthermore, the seedlings were foliar sprayed with addition of $0.5 \mu \mathrm{M}$ salicylic acid once. After 3-week experimental period, leaves of seedlings were harvested and lyophilized for analysis.

\section{Experimental design}

The experimental groups with their abbreviations were represented as follows:

T-C: Pistacia terebinthus (T)-Control

T+SA: Pistacia terebinthus (T)+Salicylic acid (SA)

$\mathbf{T}+\mathbf{C d}_{1}$ : Pistacia terebinthus $(\mathrm{T})+\mathrm{Cadmium}: 50 \mu \mathrm{M}\left(\mathrm{Cd}_{1}\right)$

$\mathbf{T}+\mathbf{C d}_{2}$ : Pistacia terebinthus $(\mathrm{T})+\mathrm{Cadmium}: 100 \mu \mathrm{M}\left(\mathrm{Cd}_{2}\right)$

$\mathbf{T}+\mathbf{C d}_{1}+$ SA: Pistacia terebinthus $(\mathrm{T})+$ Cadmium:50 $\mu \mathrm{M}\left(\mathrm{Cd}_{1}\right)+$ Salicylic acid (SA)

$\mathbf{T}+\mathbf{C d} \mathbf{d}_{2}+$ SA: Pistacia terebinthus $(\mathrm{T})+$ Cadmium: $100 \mu \mathrm{M}\left(\mathrm{Cd}_{2}\right)+$ Salicylic acid (SA)

$\mathbf{K}+\mathbf{C}$ : Pistacia khinjuk (K)-Control

K+SA: Pistacia khinjuk (K)+Salicylic acid (SA)

$\mathbf{K}+\mathbf{C d}_{1}$ : Pistacia khinjuk $(\mathrm{K})+$ Cadmium:50 $\mu \mathrm{M}\left(\mathrm{Cd}_{1}\right)$

$\mathbf{K}+\mathbf{C d}_{2}$ : Pistacia khinjuk (K)+Cadmium: $100 \mu \mathrm{M}\left(\mathrm{Cd}_{2}\right)$

$\mathbf{K}+\mathbf{C d} \mathbf{d}_{\mathbf{1}}+\mathbf{S A}$ : Pistacia khinjuk $(\mathrm{K})+$ Cadmium:50 $\mu \mathrm{M}\left(\mathrm{Cd}_{1}\right)+$ Salicylic acid (SA)

$\mathbf{K}+\mathbf{C d}_{\mathbf{2}}+\mathbf{S A}$ : Pistacia khinjuk $(\mathrm{K})+$ Cadmium:100 $\mu \mathrm{M}\left(\mathrm{Cd}_{2}\right)+$ Salicylic acid (SA)

$\mathbf{V}+\mathbf{C}$ : Pistacia vera $(\mathrm{V})-$ Control

V+SA: Pistacia vera $(\mathrm{V})+$ Salicylic acid (SA)

V+SA: Pistacia vera (V)+Salicylic acid (SA)

V+Cd1: Pistacia vera $(\mathrm{V})+\mathrm{Cadmium}: 50 \mu \mathrm{M}\left(\mathrm{Cd}_{1}\right)$

$\mathbf{V}+\mathbf{C d}_{2}$ : Pistacia vera $(\mathrm{V})+\mathrm{Cadmium}: 100 \mu \mathrm{M}\left(\mathrm{Cd}_{2}\right)$

$\mathbf{V}+\mathbf{C d}_{\mathbf{1}}+\mathbf{S A}$ : Pistacia vera $(\mathrm{V})+\mathrm{Cadmium}: 50 \mu \mathrm{M}\left(\mathrm{Cd}_{1}\right)+$ Salicylic acid (SA)

$\mathbf{V}+\mathbf{C d}_{2}+\mathbf{S A}$ : Pistacia vera $(\mathrm{V})+$ Cadmium: $100 \mu \mathrm{M}\left(\mathrm{Cd}_{2}\right)+$ Salicylic acid (SA) 


\section{Preparation of plant samples for mineral content}

First of all, the plant samples were cleaned and washed by deionized water, later air dried. Pre-dried samples were de-moisturized at $70^{\circ} \mathrm{C}$ for $48 \mathrm{~h}$ in an oven and ground for chemical analysis. $0.2 \mathrm{~g}$ of ground samples was placed into burning cup, $5 \mathrm{ml} \mathrm{HNO}_{3} 65 \%$ (Merck, Darmstadt, Germany) and $2 \mathrm{ml} \mathrm{H}_{2} \mathrm{O}_{2} 30 \%$ (Merck, Darmstadt, Germany) were added immediately. After incinerating in a HP-500CEM MARS 5 microwave (crop. Mathews NC, USA) at $200^{\circ} \mathrm{C}$, the solution was cooled at room temperature for 45 minutes. The extracts were passed through a filter paper and the filtrates were collected by high-deionized water in a $20 \mathrm{ml}$ of polyethylene bottles and kept at $4^{\circ} \mathrm{C}$ in laboratory for ICP-AES analysis. Each sample was analyzed in triplicate. Phosphorus (P) and nitrogen $(\mathrm{N})$ content were determined by vanadomolybdate method (Chapman and Pratt, 1961) and modified Kjeldahl method (Kacar and Inal, 2008) while K, Ca, Mg, S, P, Fe, $\mathrm{Zn}, \mathrm{Mo}, \mathrm{Mn}$ and $\mathrm{Cu}$ were ascertained by Inductively coupled plasma-optical emission spectrometry (ICP-OES). For all analytical works, distilled-deionized water was used. All the glassware and polyethylene bottles were attentively leached with $2-4 \% \mathrm{HCl}$ and rinsed through deionized water for three times. Merck standards (R1 and R2 groups) were used as analytical reagent grade chemicals.

\section{Statistical analysis}

Three replications were used for each treatment. Data were expressed as mean. The means were compared using the one-way ANOVA followed by Duncan's multiple range tests. The differences between individual means were considered to be significant at $p<0.05$. Moreover, a principal component analysis (PCA) was performed in order to discriminate between pistachio species exposed to the different treatments on the basis of the macro and micro elements identified along with the study. Also, correlation coefficients were calculated on the basis of the elements at different plant species in order to determine whether correlation coefficients among elements are the same or not. All analyses were performed using XLSTAT and SPSS.

\section{Results}

To assess the plausible roles of salicylic acid in reducing cadmium toxicity in P. terebinthus $(\mathrm{T})$, P. khinjuk $(\mathrm{K})$ and $P$. vera $(\mathrm{V})$ plants, the macro and micro elements and protein contents were investigated in pistachio seedlings foliar treated with $0.5 \mu \mathrm{M}$ SA under $50\left(\mathrm{Cd}_{1}\right)$ and $100\left(\mathrm{Cd}_{2}\right) \mu \mathrm{M}$ conditions, respectively.

The results of the study were analyzed using one-way variance analysis (Tables 1-2), multiple linear regression analysis (Tables 3-13), correlation analysis (Tables 14-16) and principal component analysis (Figs. 1-4) in order to define, discriminate and clarify the differences between the rootstocks of pistachio.

\section{Nitrogen $(N)$ content}

Cadmium (Cd) and salicylic acid separately caused a decrease of $\mathrm{N}$ in leaves of $P$. terebinthus but $\mathrm{N}$ content increased by the combination of cadmium concentrations with salicylic acid. However, the effects of $\mathrm{Cd}_{2}+$ and $\mathrm{Cd}_{2}+\mathrm{SA}$ were only significant ( $p=0.000$ and $p=0.004$, respectively) according to the multiple linear regression analysis. As a result of multiple linear regression analysis, which is thought to have an effect on the accumulation of $\mathrm{N}$, the variables including $\mathrm{SA}, \mathrm{Cd}_{1}+, \mathrm{Cd}_{1}+\mathrm{SA}, \mathrm{Cd}_{2}+$ and 
$\mathrm{Cd}_{2}+\mathrm{SA}$ exhibited significant effects $\left(\mathrm{R}^{2}=0.897 . \mathrm{p}<0.005\right)$. Moreover, $\mathrm{Cd}_{1}+(\mathrm{p}=0.000)$, $\mathrm{Cd}_{2}+(\mathrm{p}=0.000)$ treatments, SA $(\mathrm{p}=0.000), \mathrm{Cd}_{1}+\mathrm{SA}(\mathrm{p}=0.000)$ and $\mathrm{Cd}_{2}+\mathrm{SA}(\mathrm{p}=0.000)$ decreased $\mathrm{N}$ content in $P$. khinjuk. The significant effects of applications were also exhibited with the regression analysis $\left(\mathrm{R}^{2}=0.955, \mathrm{p}<0.005\right)$. For $P$. vera, $\mathrm{SA}$ alone $(\mathrm{p}=0.146)$ and its interaction with $\mathrm{Cd}_{1}+(\mathrm{p}=0.006)$ and $\mathrm{Cd}_{2}+(\mathrm{p}=0.315)$ caused a decline in leaf $\mathrm{N}$ content. Regression analysis exhibited non-significant effect of applications together $\left(\mathrm{R}^{2}=0.614, \mathrm{p}<0.005\right)$ (Table 3$)$. Singh and Usha (2003) reported a decline in the nitrogen content in leaves of wheat seedlings treated with salicylic acid.

\section{Phosphorus (P) content}

A quantitatively increment in leaf $P$ content of $P$. terebinthus was observed with all treatments. However, the effects of SA $(p=0.000)$ and $\mathrm{Cd}_{2}+\mathrm{SA}(\mathrm{p}=0.001)$ were deemed as significant according to the multiple linear regression analysis. However, a quantitatively decline in leaf $\mathrm{P}$ content of $P$. khinjuk was recorded under $\mathrm{SA}+$ $(\mathrm{p}=0.146), \mathrm{Cd}_{1}+\mathrm{SA}(\mathrm{p}=0.006) \mathrm{Cd}_{2}+\mathrm{SA}(\mathrm{p}=0.315)$, suggesting the adverse influence of SA on P content for P. khinjuk. The similar results regarding $\mathrm{P}$ content were observed for P. vera in response to treatments (Table 4). In the study by Wang et al. (2011), SA alone increased the $\mathrm{P}$ concentration whereas $\mathrm{Pb}$ decreased the concentration. $50 \mu \mathrm{M} \mathrm{Pb}-$ induced decrease was improved by the addition of $10 \mu \mathrm{M} \mathrm{SA}$.

\section{Potassium (K) content}

Augmented and significant changes were recorded except SA alone treatment, of which effects were not significant $(\mathrm{p}=0.421)$ for $\mathrm{K}$ content of $P$. terebinthus. For $P$. khinjuk, all treatments adversely affected the $\mathrm{K}$ content in leaves. Of the treatments, $\mathrm{SA}+(\mathrm{p}=0.884)$ and $\mathrm{Cd}_{1}+(\mathrm{p}=0.356)$ caused decreases in $\mathrm{K}$ content for $P$. vera but the changes were not significant. The interaction of SA and $\mathrm{Cd}_{1}+$ increased the content $(\mathrm{p}=0.011)$. Interestingly, whereas $\mathrm{Cd}_{2}+$ significantly increased the content, $\mathrm{Cd}_{2}+\mathrm{SA}$ interaction did not cause a significant change $(p=0.259)$, proposing the influence of SA on the activities of cadmium treatments (Table 5).

The treatments of excessive Mn, salicylic acid and both did not significantly affect $\mathrm{K}$ content in leaves (Shi and Zhu, 2008).

\section{Calcium (Ca) content}

Attenuated changes were observed with the treatments except SA+, of which effect was not significant $(\mathrm{p}=0.136)$. Interaction of $\mathrm{Cd}_{1}+\mathrm{SA}$ decreased $\mathrm{Ca}$ content $(\mathrm{p}=0.000)$ whereas $\mathrm{Cd}_{2}+\mathrm{SA}$ caused an increase in Ca content $(\mathrm{p}=0.011)$ for P. terebinthus. We noted the decreases in $\mathrm{Ca}$ content in leaves of $P$. khinjuk under all treatments except $\mathrm{Cd}_{2}+\mathrm{SA}$, which was not significant $(\mathrm{p}=0.346)$. The quantitatively attenuated changes were significant for other treatments, whereas SA coupled with $\mathrm{Cd}_{1}+$ decreased the content; SA suppressed the attenuated change in Ca content by $\mathrm{Cd}_{2}+$.

The similar responses against treatments were observed by $P$. vera. SA improved quantitatively the adverse effects of $\mathrm{Cd}_{1}+$ and $\mathrm{Cd}_{2}+$ treatments $(\mathrm{p}=0.201$ and $\mathrm{p}=0.570$, respectively) (Table 6). In the study by Shi and Zhu (2008), salicylic acid increased Ca content and excessive Mn decreased the content but the addition of SA significantly increased $\mathrm{Ca}$ concentration under excess $\mathrm{Mn}$ condition. Furthermore, $\mathrm{Pb}$ treatments and SA alone treatment induced an increase in $\mathrm{Ca}$ concentration whereas addition of SA significantly decreased under $\mathrm{Pb}$ condition (Wang et al., 2011). 
Table 1. Macro element contents in leaves of pistachio species under different treatments

\begin{tabular}{|c|c|c|c|c|c|c|c|c|c|c|c|c|}
\hline Groups & $\mathbf{N}$ & Change (\%) & $\mathbf{P}$ & Change (\%) & $\mathbf{K}$ & Change (\%) & $\mathrm{Ca}$ & Change (\%) & Mg & Change (\%) & Protein & Change (\%) \\
\hline T-C & $1.77 \mathrm{fgh}$ & 0.00 & $2.07 \mathrm{de}$ & 0.00 & $0.58 \mathrm{gh}$ & 0.00 & $1.51 \mathrm{a}$ & 0.00 & $0.22 \mathrm{de}$ & 0.00 & 10.96 ef & 0.00 \\
\hline $\mathrm{T}+\mathrm{SA}$ & $1.74 \mathrm{hg}$ & -1.69 & $4.5 \mathbf{a}$ & 117.39 & $0.52 \mathrm{~h}$ & -10.3 & $1.61 \mathbf{a}$ & 6.62 & $0.32 \mathbf{a b c}$ & 45.45 & 10.78 ef & -1.64 \\
\hline $\mathrm{T}+\mathrm{Cd} 1$ & $1.70 \mathrm{ih}$ & -3.95 & $2.09 \mathrm{de}$ & 0.97 & 0.82 be & 41.38 & $1.06 \mathrm{def}$ & -29.80 & 0.16 ef & -27.27 & $10.60 f$ & -3.28 \\
\hline $\mathrm{T}+\mathrm{Cd} 2$ & $1.51 \mathbf{j}$ & -14.69 & $2.24 \mathrm{de}$ & 8.21 & 0.78 cf & 34.48 & $1.08 \mathbf{c f}$ & -28.48 & 0.17 ef & -22.73 & $9.38 \mathbf{h}$ & -14.42 \\
\hline $\mathrm{T}+\mathrm{Cd} 1+\mathrm{SA}$ & $1.78 \mathrm{fgh}$ & 0.56 & $2.23 \mathrm{de}$ & 7.73 & $0.9 \mathbf{b c}$ & 55.17 & 1 def & -33.77 & $0.26 \mathrm{bcd}$ & 18.18 & $11.12 \mathrm{de}$ & 1.46 \\
\hline $\mathrm{T}+\mathrm{Cd} 2+\mathrm{SA}$ & $1.92 \mathrm{de}$ & 8.47 & $3.45 \mathbf{b}$ & 66.67 & $0.94 \mathbf{b}$ & 62.07 & $1.3 \mathbf{b}$ & -13.91 & $0.2 \mathrm{def}$ & -9.09 & $12.00 \mathrm{c}$ & 9.49 \\
\hline $\mathrm{K}+\mathrm{C}$ & $2.07 \mathrm{~b}$ & 0.00 & $2.38 \mathrm{de}$ & 0.00 & $1.07 \mathbf{a}$ & 0.00 & $1.16 \mathrm{bcd}$ & 0.00 & $0.22 \mathrm{de}$ & 0.00 & $12.84 \mathbf{b}$ & 0.00 \\
\hline $\mathrm{K}+\mathrm{SA}$ & $1.46 \mathbf{j}$ & -29.47 & $2.03 \mathrm{de}$ & -14.71 & $0.94 \mathbf{b}$ & -12.1 & $0.92 \mathrm{fgh}$ & -20.69 & 0.17 ef & -22.73 & $9.03 \mathbf{h}$ & -29.67 \\
\hline $\mathrm{K}+\mathrm{Cd} 1$ & $1.62 \mathbf{i}$ & -21.74 & $3.2 \mathrm{bc}$ & 34.45 & $1.17 \mathbf{a}$ & 9.35 & $0.95 \mathrm{eh}$ & -18.10 & $0.13 \mathrm{f}$ & -40.91 & $10.08 \mathrm{~g}$ & -21.50 \\
\hline $\mathrm{K}+\mathrm{Cd} 2$ & $1.84 \mathrm{def}$ & -11.11 & $2.55 \mathrm{~cd}$ & 7.14 & $0.93 \mathbf{b}$ & -13.1 & $0.8 \mathrm{gh}$ & -31.03 & 0.18 def & -18.18 & $11.48 \mathrm{~d}$ & -10.59 \\
\hline $\mathrm{K}+\mathrm{Cd} 1+\mathrm{SA}$ & $1.75 \mathrm{fgh}$ & -15.46 & $1.64 \mathrm{e}$ & -31.09 & $0.66 \mathrm{fg}$ & -38.3 & $0.82 \mathrm{gh}$ & -29.31 & $0.13 \mathrm{f}$ & -40.91 & 10.95 ef & -14.72 \\
\hline $\mathrm{K}+\mathrm{Cd} 2+\mathrm{SA}$ & 1.83 efg & -11.59 & $2.14 \mathrm{de}$ & -10.08 & $0.87 \mathrm{bcd}$ & -18.69 & $1.23 \mathrm{bc}$ & 6.03 & $0.23 \mathrm{de}$ & 4.55 & $11.47 \mathrm{~d}$ & -10.67 \\
\hline $\mathrm{V}+\mathrm{C}$ & $1.96 \mathbf{c}$ & 0.00 & $1.82 \mathrm{de}$ & 0.00 & 0.74 def & 0.00 & 1.11 cde & 0.00 & 0.25 cde & 0.00 & $12.18 \mathrm{c}$ & 0.00 \\
\hline $\mathrm{V}+\mathrm{SA}$ & $1.63 \mathbf{i}$ & -16.84 & $2.11 \mathrm{de}$ & 15.93 & 0.73 ef & -1.35 & $1.48 \mathbf{a}$ & 33.33 & $0.37 \mathbf{a}$ & 48.00 & $10.08 \mathrm{~g}$ & -17.24 \\
\hline $\mathrm{V}+\mathrm{Cd} 1$ & $1.93 \mathrm{~d}$ & -1.53 & $2.15 \mathrm{de}$ & 18.13 & 0.69 efg & -6.76 & $0.79 \mathbf{h}$ & -28.83 & $0.22 \mathrm{de}$ & -12.00 & $12.00 \mathrm{c}$ & -1.48 \\
\hline $\mathrm{V}+\mathrm{Cd} 2$ & $2.82 \mathrm{a}$ & 43.88 & $1.89 \mathrm{de}$ & 3.85 & $0.93 \mathbf{b}$ & 25.68 & 0.96 efg & -13.51 & $0.22 \mathrm{de}$ & -12.00 & $17.60 \mathbf{a}$ & 44.50 \\
\hline $\mathrm{V}+\mathrm{Cd} 1+\mathrm{SA}$ & $1.92 \mathrm{de}$ & -2.04 & $2.23 \mathrm{de}$ & 22.53 & $0.9 \mathbf{b c}$ & 21.62 & $1.0 \mathrm{def}$ & -9.91 & $0.26 \mathrm{bcd}$ & 4.00 & $12.00 \mathrm{c}$ & -1.48 \\
\hline $\mathrm{V}+\mathrm{Cd} 2+\mathrm{SA}$ & $1.94 \mathrm{c}$ & -1.02 & $3.34 \mathbf{b}$ & 83.52 & 0.81 be & 9.46 & $1.06 \mathrm{def}$ & -4.50 & $0.34 \mathbf{a b}$ & 36.00 & $12.17 \mathrm{c}$ & -0.08 \\
\hline
\end{tabular}

Table 2. Micro element contents in leaves of pistachio species under different treatments

\begin{tabular}{|c|c|c|c|c|c|c|c|c|c|c|}
\hline Groups & $\mathbf{Z n}$ & Change (\%) & Mn & Change (\%) & $\mathrm{Cu}$ & Change (\%) & $\mathrm{Fe}$ & Change (\%) & Cd & Change (\%) \\
\hline $\mathrm{T}-\mathrm{C}$ & $11.66 \mathrm{ad}$ & 0.00 & $1.61 \mathrm{fg}$ & 0.00 & $0.21 \mathbf{e}$ & 0.00 & 46.6cde & 0.00 & $0.31 \mathbf{d}$ & 0.00 \\
\hline $\mathrm{T}+\mathrm{SA}$ & $5.22 \mathrm{dh}$ & -55.23 & $1.47 \mathrm{~g}$ & -8.70 & $1.12 \mathbf{a e}$ & 433.33 & $42.2 \mathrm{ed}$ & -9.44 & $0.30 \mathrm{~d}$ & -3.23 \\
\hline $\mathrm{T}+\mathrm{Cd} 1$ & $0.67 \mathbf{h}$ & -94.25 & 4.48 cde & 178.26 & 0.91 cde & 333.33 & $55.36 \mathrm{be}$ & 18.80 & $0.41 \mathbf{a b}$ & 32.26 \\
\hline $\mathrm{T}+\mathrm{Cd} 2$ & $0.61 \mathrm{gh}$ & -94.77 & $4.9 \mathrm{cde}$ & 204.35 & $1.09 \mathrm{be}$ & 419.05 & $59.7 \mathrm{bcd}$ & 28.11 & $0.41 \mathbf{a b}$ & 32.26 \\
\hline $\mathrm{T}+\mathrm{Cd} 1+\mathrm{SA}$ & $3.19 \mathrm{fgh}$ & -72.64 & $3.85 \mathrm{dg}$ & 139.13 & $1.33 \mathrm{ad}$ & 533.33 & 46.4cde & -0.43 & $0.46 \mathbf{a}$ & 48.39 \\
\hline $\mathrm{T}+\mathrm{Cd} 2+\mathrm{SA}$ & $8.68 \mathrm{bf}$ & -25.56 & 2.45 efg & 52.17 & $0.35 \mathrm{de}$ & 66.67 & $72.7 \mathbf{a b}$ & 56.01 & $0.39 \mathrm{bc}$ & 25.81 \\
\hline $\mathrm{K}+\mathrm{C}$ & $16.59 \mathbf{a}$ & 0.00 & $4.76 \mathrm{cde}$ & 0.00 & $1.12 \mathbf{a e}$ & 0.00 & $66.2 \mathrm{~b}$ & 0.00 & $0.3 \mathbf{d}$ & 0.00 \\
\hline $\mathrm{K}+\mathrm{SA}$ & $13.9 \mathbf{a b}$ & -16.21 & $4.1 \mathbf{c - f}$ & -13.87 & $1.37 \mathbf{a d}$ & 22.32 & $69.37 \mathbf{b}$ & 4.79 & $0.4 \mathbf{a b}$ & 33.33 \\
\hline $\mathrm{K}+\mathrm{Cd} 1$ & $10.82 \mathbf{a e}$ & -34.78 & $6.48 \mathrm{bc}$ & 36.13 & $1.82 \mathbf{a b c}$ & 62.50 & $61.6 \mathbf{b c}$ & -6.95 & $0.34 \mathbf{c d}$ & 13.33 \\
\hline $\mathrm{K}+\mathrm{Cd} 2$ & $7.6 \mathrm{bg}$ & -54.19 & 2.73 efg & -42.65 & 0.91 cde & -18.75 & $64.27 \mathbf{b}$ & -2.92 & $0.41 \mathbf{a b}$ & 36.67 \\
\hline $\mathrm{K}+\mathrm{Cd} 1+\mathrm{SA}$ & $11.66 \mathbf{a d}$ & -29.72 & $5.7 \mathrm{~cd}$ & 19.75 & $1.19 \mathrm{ae}$ & 6.25 & $85.4 \mathbf{a}$ & 29.00 & $0.3 \mathbf{d}$ & 0.00 \\
\hline $\mathrm{K}+\mathrm{Cd} 2+\mathrm{SA}$ & $12.18 \mathbf{a b c}$ & -26.58 & 4.17 cde & -12.39 & $2.03 \mathbf{a b}$ & 81.25 & $61.2 \mathrm{bc}$ & -7.55 & $0.40 \mathbf{a b}$ & 33.33 \\
\hline $\mathrm{V}+\mathrm{C}$ & $4.45 \mathrm{eh}$ & 0.00 & $1.47 \mathrm{~g}$ & 0.00 & $1.47 \mathbf{a b c}$ & 0.00 & $59.6 \mathrm{bcd}$ & 0.00 & $0.42 \mathbf{a b}$ & 0.00 \\
\hline $\mathrm{V}+\mathrm{SA}$ & $5.99 \mathrm{ch}$ & 34.61 & $14.56 \mathbf{a}$ & 890.48 & $1.89 \mathbf{a b c}$ & 28.57 & 45.73 cde & -23.27 & $0.40 \mathbf{a b}$ & -4.76 \\
\hline $\mathrm{V}+\mathrm{Cd} 1$ & $4.38 \mathrm{eh}$ & -1.57 & $3.33 \mathrm{dg}$ & 126.53 & $1.82 \mathbf{a b c}$ & 23.81 & $39.8 \mathbf{e}$ & -33.22 & $0.41 \mathbf{a b}$ & -2.38 \\
\hline $\mathrm{V}+\mathrm{Cd} 2$ & $9.87 \mathrm{be}$ & 121.80 & 3.15 efg & 114.29 & $2.14 \mathbf{a}$ & 45.58 & $43.6 \mathrm{ed}$ & -26.85 & $0.40 \mathbf{a b}$ & -4.76 \\
\hline $\mathrm{V}+\mathrm{Cd} 1+\mathrm{SA}$ & $3.19 \mathrm{fgh}$ & -28.31 & $3.85 \mathrm{dg}$ & 161.90 & $1.33 \mathbf{a d}$ & -9.52 & $46.4 \mathrm{cde}$ & -22.15 & $0.38 \mathbf{b c}$ & -9.52 \\
\hline $\mathrm{V}+\mathrm{Cd} 2+\mathrm{SA}$ & $7.63 \mathrm{bg}$ & 71.46 & $8.02 \mathbf{b}$ & 445.58 & 0.95 cde & -35.37 & $56.2 \mathrm{be}$ & -5.70 & $0.42 \mathbf{a b}$ & 0.00 \\
\hline
\end{tabular}

APPLIED ECOLOGY AND ENVIRONMENTAL RESEARCH 17(3): 7113-7133.

http://www.aloki.hu • ISSN 15891623 (Print) • ISSN 17850037 (Online)

DOI: http://dx.doi.org/10.15666/aeer/1703 71137133

๑ 2019, ALÖKI Kft., Budapest, Hungary 
Table 3. $N$ contents in leaves of pistachio species under different treatments

\begin{tabular}{|c|c|c|c|c|c|c|c|c|c|}
\hline \multirow{2}{*}{$\mathbf{N}$} & \multicolumn{3}{|c|}{ P. terebinthus } & \multicolumn{3}{|c|}{ P. khinjuk } & \multicolumn{3}{|c|}{ P. vera } \\
\hline & B & Mean Dif. & Sig. & B & Mean Dif. & Sig. & B & Mean Dif. & Sig. \\
\hline (Constant) & 1.768 & & .000 & 2.070 & & .000 & 1.964 & & .000 \\
\hline SA+ & -.028 & .03000 & .511 & -.610 & 61000 & .000 & -.336 & .33000 & .000 \\
\hline $\mathrm{Cd}_{1}+$ & -.066 & .07000 & .137 & -.452 & .45000 & .000 & -.038 & .03000 & .376 \\
\hline $\mathrm{Cd}_{2}+$ & -.262 & .26000 & .000 & -.228 & .23000 & .000 & .858 & -.86000 & .000 \\
\hline $\mathrm{Cd}_{1}+\mathrm{SA}$ & .008 & -.01000 & .850 & -.322 & .32000 & .000 & -.048 & .04000 & .268 \\
\hline $\mathrm{Cd}_{2}+\mathrm{SA}$ & .148 & -.15000 & .004 & -.238 & .24000 & .000 & -.020 & .02000 & .637 \\
\hline $\mathrm{R}^{2}$ & \multicolumn{3}{|c|}{.897} & \multicolumn{3}{|c|}{.955} & \multicolumn{3}{|c|}{.988} \\
\hline
\end{tabular}

Table 4. $P$ contents in leaves of pistachio species under different treatments

\begin{tabular}{|c|c|c|c|c|c|c|c|c|c|}
\hline \multirow{2}{*}{$\mathbf{P}$} & \multicolumn{3}{|c|}{ P. terebinthus } & \multicolumn{3}{|c|}{ P. khinjuk } & \multicolumn{3}{|c|}{ P. vera } \\
\hline & $\mathbf{B}$ & Mean Dif. & Sig. & B & Mean Dif. & Sig. & B & Mean Dif. & Sig. \\
\hline (Constant) & 2.070 & & .000 & 2.380 & & .000 & 1.821 & & .000 \\
\hline $\mathrm{SA}+$ & 2.428 & -2.430 & .000 & -.347 & .34667 & .146 & .289 & -.2900 & .482 \\
\hline $\mathrm{Cd}_{1}+$ & .018 & -.0166 & .956 & .820 & -.8200 & .003 & .324 & -.3266 & .432 \\
\hline $\mathrm{Cd}_{2}+$ & .166 & -.1666 & .609 & .174 & -.1700 & .452 & .074 & -.0733 & .856 \\
\hline $\mathrm{Cd}_{1}+\mathrm{SA}$ & .158 & -.1600 & .627 & -.743 & .7400 & .006 & .407 & -.4100 & .328 \\
\hline $\mathrm{Cd}_{2}+\mathrm{SA}$ & 1.383 & -1.380 & .001 & -.234 & .2333 & .315 & 1.514 & -1.516 & .003 \\
\hline $\mathrm{R}^{2}$ & \multicolumn{3}{|c|}{.893} & \multicolumn{3}{|c|}{.825} & \multicolumn{3}{|c|}{.614} \\
\hline
\end{tabular}

Table 5. $K$ contents in leaves of pistachio species under different treatments

\begin{tabular}{|c|c|c|c|c|c|c|c|c|c|}
\hline \multirow{2}{*}{$\mathbf{K}$} & \multicolumn{3}{|c|}{ P. terebinthus } & \multicolumn{3}{|c|}{ P. khinjuk } & \multicolumn{3}{|c|}{ P. vera } \\
\hline & B & Mean Dif. & Sig. & B & Mean Dif. & Sig. & B & Mean Dif. & Sig. \\
\hline (Constant) & .581 & & .000 & 1.070 & & .000 & .745 & & .000 \\
\hline $\mathrm{SA}+$ & -.057 & .05667 & .421 & -.133 & .1333 & .024 & -.008 & .00667 & .884 \\
\hline $\mathrm{Cd}_{1}+$ & .243 & -.2433 & .004 & .103 & -.1000 & .069 & -.050 & .05000 & .356 \\
\hline $\mathrm{Cd}_{2}+$ & .196 & -.2000 & .015 & -.145 & .1400 & .016 & .183 & -.18333 & .005 \\
\hline $\mathrm{Cd}_{1}+\mathrm{SA}$ & .322 & -.3233 & .001 & -.407 & .4100 & .000 & .158 & -.16000 & .011 \\
\hline $\mathrm{Cd}_{2}+\mathrm{SA}$ & .357 & -.3600 & .000 & -.200 & .2000 & .002 & .062 & -.06667 & .259 \\
\hline $\mathrm{R}^{2}$ & & .836 & & & .906 & & & .731 & \\
\hline
\end{tabular}

Table 6. Ca contents in leaves of pistachio species under different treatments

\begin{tabular}{|c|c|c|c|c|c|c|c|c|c|}
\hline \multirow{2}{*}{ Ca } & \multicolumn{3}{|c|}{ P. terebinthus } & \multicolumn{3}{|c|}{ P. khinjuk } & \multicolumn{3}{|c|}{ P. vera } \\
\hline & B & Mean Dif. & Sig. & B & Mean Dif. & Sig. & B & Mean Dif. & Sig. \\
\hline (Constant) & 1.509 & & .000 & 1.160 & & .000 & 1.110 & & .000 \\
\hline $\mathrm{SA}+$ & .109 & -.1066 & .136 & -.238 & .23667 & .005 & .370 & -.36667 & .001 \\
\hline $\mathrm{Cd}_{1}+$ & -.449 & .45000 & .137 & -.211 & .21000 & .010 & -.321 & .32000 & .002 \\
\hline $\mathrm{Cd}_{2}+$ & -.430 & .43000 & .000 & -.356 & .35667 & .000 & -.151 & .15000 & .085 \\
\hline $\mathrm{Cd}_{1}+\mathrm{SA}$ & -.508 & .50667 & .000 & -.340 & .33667 & .000 & -.109 & .10667 & .201 \\
\hline $\mathrm{Cd}_{2}+\mathrm{SA}$ & -.205 & .20667 & .011 & .068 & -.0700 & .346 & -.047 & .04667 & .570 \\
\hline $\mathrm{R}^{2}$ & & .923 & & & .842 & & & .873 & \\
\hline
\end{tabular}

\section{Magnesium (Mg) content}

Mg content decreased with $\mathrm{Cd}_{1}+(\mathrm{p}=0.101)$ and $\mathrm{Cd}_{2}+(\mathrm{p}=0.101)$ treatments but the content was quantitatively but not significantly improved with $\mathrm{SA}+(\mathrm{p}=0.016)$ alone or interaction with $\mathrm{Cd}_{1}+(\mathrm{p}=0.285)$ and $\mathrm{Cd}_{2}+(\mathrm{p}=0.579)$ in P. terebinthus. All treatments 
except $\mathrm{Cd}_{2}+\mathrm{SA}(\mathrm{p}=0.767)$ caused significantly decreases in $\mathrm{Mg}$ content in P. khinjuk. Treatments including $\mathrm{SA}+(\mathrm{p}=0.029), \mathrm{Cd}_{1}+\mathrm{SA}(\mathrm{p}=0.762)$ and $\mathrm{Cd}_{2}+\mathrm{SA}(\mathrm{p}=0.089)$ improved the $\mathrm{Mg}$ content whereas $\mathrm{Cd}_{1}+(\mathrm{p}=0.603)$ and $\mathrm{Cd}_{2}+(\mathrm{p}=0.582)$ decreased the content of $\mathrm{Mg}$ in P. vera (Table 7). For the case in cucumber (Shi and Zhu, 2008), the treatment SA did not significantly influence the $\mathrm{Mg}$ concentration but excess $\mathrm{Mn}$ significantly decreased the $\mathrm{Mg}$ concentration in leaf tissues. After, addition of SA significantly improved the concentration.

Table 7. Mg contents in leaves of pistachio species under different treatments

\begin{tabular}{|c|c|c|c|c|c|c|c|c|c|}
\hline \multirow{2}{*}{ Mg } & \multicolumn{3}{|c|}{ P. terebinthus } & \multicolumn{3}{|c|}{ P. khinjuk } & \multicolumn{3}{|c|}{ P. vera } \\
\hline & B & Mean Dif. & Sig. & B & Mean Dif. & Sig. & B & Mean Dif. & Sig. \\
\hline (Constant) & .224 & & .000 & .224 & & .000 & .247 & & .000 \\
\hline $\mathrm{SA}+$ & .096 & -.09667 & .016 & -.054 & .05333 & .000 & .124 & -.12333 & .029 \\
\hline $\mathrm{Cd}_{1}+$ & -.061 & .06000 & .101 & -.092 & .09000 & .000 & -.027 & .02667 & .603 \\
\hline $\mathrm{Cd}_{2}+$ & -.050 & .05000 & .168 & -.044 & .04333 & .001 & -.028 & .02667 & .582 \\
\hline $\mathrm{Cd}_{1}+\mathrm{SA}$ & .038 & -.04000 & .285 & -.094 & .09333 & .000 & .016 & -.01667 & .762 \\
\hline $\mathrm{Cd}_{2}+\mathrm{SA}$ & -.020 & .02000 & .579 & .003 & -.00333 & .767 & .093 & -.09333 & .089 \\
\hline $\mathrm{R}^{2}$ & & .711 & & & .938 & & & .577 & \\
\hline
\end{tabular}

\section{Iron (Fe) content}

$\mathrm{SA}+$ alone $(\mathrm{p}=0.524)$ and its interaction with $\mathrm{Cd}_{1}\left(\mathrm{Cd}_{1}+\mathrm{SA} ; \mathrm{p}=0.976\right)$ treatments decreased $\mathrm{Fe}$ content whereas $\mathrm{Cd}_{1}+(\mathrm{p}=0.221)$ and $\mathrm{Cd}_{2}+(\mathrm{p}=0.078)$ increased the content. The interaction of SA with higher concentration of cadmium $\left(\mathrm{Cd}_{2}+\right)$ approximately doubled the content in P. terebinthus $(\mathrm{p}=0.002)$. Interestingly, $P$. khinjuk exhibited converse responses against treatments in comparison with $P$. terebinthus. Herewith, $\mathrm{SA}+(\mathrm{p}=0.713)$ and $\mathrm{Cd}_{1}+\mathrm{SA}(\mathrm{p}=0.040)$ increased Fe content whereas $\mathrm{Cd}_{1}+$ and $\mathrm{Cd}_{2}+$ caused quantitively but non-significant changes $(\mathrm{p}=0.590$ and $\mathrm{p}=0.818$, respectively). On the other hand, treatments including $\mathrm{SA}+\left(\mathrm{p}=0.072, \mathrm{Cd}_{1}+(\mathrm{p}=0.015)\right.$ and $\mathrm{Cd}_{2}+(\mathrm{p}=0.042)$ adversely affected Fe content but the adverse effects of $\mathrm{Cd} 1+$ and $\mathrm{Cd} 2+$ treatments were quantitively but non-significant improved with $\mathrm{SA}+(\mathrm{p}=0.085$ and $\mathrm{p}=0.636$, respectively) $P$. vera. According to the multiple linear regression analysis, treatments were not significant for $P$. khinjuk and $P$. vera $\left(\mathrm{R}^{2}=0.494, \mathrm{p}>0.05 ; \mathrm{R}^{2}=0.499\right.$, $\mathrm{p}>0.05$, respectively) (Table 8). Of the heavy metals, excess treatment of $\mathrm{Mn}$ and SA diminished $\mathrm{Fe}$ concentration in leaves of cucumber. However, addition of SA aggravated the decline in leaves (Shi and $\mathrm{Zhu}, 2008) . \mathrm{Pb}$ alone significantly increased the Fe concentration in comparison with control whereas SA caused a decline in the concentration. With the addition of SA, a statistically significant decrease was observed in the plants grown under $\mathrm{Pb}$ (Wang et al., 2011).

Table 8. Fe contents in leaves of pistachio species under different treatments

\begin{tabular}{c|ccc|ccc|ccc}
\hline \multirow{2}{*}{ Fe } & \multicolumn{3}{|c|}{ P. terebinthus } & \multicolumn{3}{c|}{ P. khinjuk } & \multicolumn{3}{c}{ P. vera } \\
\cline { 2 - 9 } & B & Mean Dif. & Sig. & B & Mean Dif. & Sig. & B & Mean Dif. & Sig. \\
\hline (Constant) & 46.62 & & .000 & 66.22 & & .000 & 59.57 & & .000 \\
$\mathrm{SA}+$ & -4.445 & 4.4000 & .524 & 3.150 & -3.1666 & .713 & -13.8 & 13.8666 & .072 \\
$\mathrm{Cd}_{1}+$ & 8.750 & -8.7666 & .221 & -4.62 & 4.6000 & .590 & -19.7 & 19.8000 & .015 \\
$\mathrm{Cd}_{2}+$ & 13.06 & -13.100 & .078 & -1.96 & 1.9333 & .818 & -15.9 & 16.0000 & .042 \\
$\mathrm{Cd}_{1}+\mathrm{SA}$ & -.210 & .20000 & .976 & 19.18 & -19.200 & .040 & -13.1 & 13.2000 & .085 \\
$\mathrm{Cd}_{2}+\mathrm{SA}$ & 26.11 & -26.100 & .002 & -5.04 & 5.0000 & .557 & -3.39 & 3.40000 & .636 \\
\hline $\mathrm{R}^{2}$ & \multicolumn{3}{|c|}{.698} & \multicolumn{3}{c|}{$.494(\mathrm{p}>.05)$} & & & $.499(\mathrm{p}>.05)$ \\
\hline
\end{tabular}




\section{Copper (Cu) content}

A quantitively but non-significant change was recorded for $\mathrm{Cu}$ content under all treatments in $P$. terebinthus $\left(\mathrm{R}^{2}=0.488, \mathrm{p}>0.05\right)$ but we should note that $\mathrm{Cd}_{1}+\mathrm{SA}$ increased the content once compared to $\mathrm{SA}+$ and $\mathrm{Cd}_{1}+$ whereas $\mathrm{Cd}_{2}+\mathrm{SA}$ decreased the content in comparison with $\mathrm{SA}+$ and $\mathrm{Cd}_{2}+$. Cu contents were positively influenced with the $\mathrm{SA}+$ but $\mathrm{Cd}_{1}+$ and $\mathrm{Cd}_{2}+$. However, the adverse effects of $\mathrm{Cd}_{1}+$ and $\mathrm{Cd}_{2}+$ were improved with the foliar application of SA+ for P. khinjuk.

Interestingly, $\mathrm{SA}+, \mathrm{Cd}_{1}+$ and $\mathrm{Cd}_{2}+$ increased the content but foliar application of $\mathrm{SA}$ to the plants exposed to the $\mathrm{Cd}_{1}+$ and $\mathrm{Cd}_{2}+$ diminished the content in P. vera (Table 9). Shi and Zhu (2008) reported that SA treatment did not significantly change the $\mathrm{Cu}$ concentration and excess $\mathrm{Mn}$ treatment significantly decreased the concentration. However, addition of SA did not cause any changes in the concentration. The concentration of $\mathrm{Cu}$ decreased significantly in leaves of Vallisneria natans by SA treatment. $\mathrm{Pb}$ treatment did not cause significant changes on the concentration but addition of $100 \mu \mathrm{M}$ SA significantly decreased the concentration (Wang et al., 2011).

Table 9. Cu contents in leaves of pistachio species under different treatments

\begin{tabular}{c|ccc|ccc|ccc}
\hline \multirow{2}{*}{ Cu } & \multicolumn{3}{|c|}{ P. terebinthus } & \multicolumn{3}{c|}{ P. khinjuk } & \multicolumn{3}{c}{ P. vera } \\
\cline { 2 - 9 } & B & Mean Dif. & Sig. & B & Mean Dif. & Sig. & B & Mean Dif. & Sig. \\
\hline (Constant) & .210 & & .496 & 1.120 & & .015 & 1.470 & & .000 \\
$\mathrm{SA}+$ & .910 & -.91000 & .052 & .245 & -.24666 & .670 & .420 & -.42000 & .090 \\
$\mathrm{Cd}_{1}+$ & .700 & -.70000 & .124 & .700 & -.70000 & .235 & .350 & -.35000 & .151 \\
$\mathrm{Cd}_{2}+$ & .875 & -.87666 & .061 & -.210 & .21000 & .714 & .665 & -.66667 & .013 \\
$\mathrm{Cd}_{1}+\mathrm{SA}$ & 1.120 & -1.1200 & .021 & .070 & -.07000 & .903 & -.140 & .140000 & .551 \\
$\mathrm{Cd}_{2}+\mathrm{SA}$ & .140 & -.14000 & .746 & .910 & -.91000 & .130 & -.525 & .523333 & .040 \\
\hline $\mathrm{R}^{2}$ & \multicolumn{3}{|c|}{$.488 ; \mathrm{p}>.05$} & \multicolumn{3}{c}{$.332(\mathrm{p}>.05)$} & & & .750 \\
\hline
\end{tabular}

\section{Zinc (Zn) content}

$\mathrm{Zn}$ content was significantly decreased with all treatments except $\mathrm{Cd}_{2}+\mathrm{SA}(\mathrm{p}=0.147)$ but the adverse effects of $\mathrm{Cd}_{1}+$ and $\mathrm{Cd}_{2}+$ was improved by foliar application of $\mathrm{SA}$ $(\mathrm{p}=0.001$ and $\mathrm{p}=0.147)$. The most hazardous effects on $\mathrm{Zn}$ content was more pronounced under $\mathrm{Cd}_{1}+$ and $\mathrm{Cd}_{2}+$ for $P$. terebinthus. The similar responses in accumulation of $\mathrm{Zn}$ against treatments were also recorded for $P$. khinjuk. On the other hand, $\mathrm{SA}+$ and $\mathrm{Cd}_{2}+$ caused increases in $\mathrm{Zn}$ and $\mathrm{Cd}_{1}+$ decreased the content. The contents in plants exposed to $\mathrm{Cd}_{1}+$ and $\mathrm{Cd}_{2}+$ were still decreased with foliar application of SA. We should state that the quantitative changes were not significant $\left(\mathrm{R}^{2}=0.381\right.$, $\mathrm{p}>0.05$ ) for $P$. vera (Table 10). The $\mathrm{Zn}$ concentration leaves was significantly diminished with the excess Mn treatment but the decrease was alleviated by SA addition under excess Mn treatment in cucumber. Furthermore, SA alone treatment itself did not significantly change the $\mathrm{Zn}$ concentration (Shi and $\mathrm{Zhu}, 2008$ ). $\mathrm{Pb}$ treatment did not significantly affect the $\mathrm{Zn}$ concentration. SA alone induced a decrease in the concentration (Wang et al., 2011).

\section{Manganese (Mn) content}

Mn content was adversely affected with foliar application of SA. Cadmium treatments $\left(\mathrm{Cd}_{1}+; \mathrm{p}=0.001\right.$ and $\left.\mathrm{Cd}_{2}+; \mathrm{p}=0.000\right)$ increased Mn content but the effects of treatments were suppressed with $\mathrm{SA}\left(\mathrm{Cd}_{1}+\mathrm{SA} ; \mathrm{p}=0.006\right.$ and $\left.\mathrm{Cd}_{2}+\mathrm{SA} ; \mathrm{p}=0.230\right)$ for 
P. terebinthus. SA+ and $\mathrm{Cd}_{2}+$ decreased the content whereas the lower cadmium treatment $\left(\mathrm{Cd}_{1}+\right)$ increased the content. However, $\mathrm{SA}+$ treatment caused a decrease in $\mathrm{Mn}$ content in plants exposed to $\mathrm{Cd}_{1}+$ treatment and vice-verse for the plants under $\mathrm{Cd}_{2}+$ treatment for $P$. khinjuk. All treatments positively influenced $\mathrm{Mn}$ content in P. vera (Table 11). Of the heavy metals, treatments with $50 \mu \mathrm{M} \mathrm{Pb}$ induced significant decrease of $\mathrm{Mn}$ and SA alone did not exhibit significant effects on Mn concentration. However, the decrease was alleviated by SA addition in Vallisneria natans under $\mathrm{Pb}$ treatment (Wang et al., 2011).

Table 10. Zn contents in leaves of pistachio species under different treatments

\begin{tabular}{c|ccc|ccc|ccc}
\hline \multirow{2}{*}{$Z \mathbf{n}$} & \multicolumn{3}{|c|}{ P. terebinthus } & \multicolumn{3}{c|}{ P. khinjuk } & \multicolumn{3}{c}{ P. vera } \\
\cline { 2 - 9 } & B & Mean Dif. & Sig. & B & Mean Dif. & Sig. & B & Mean Dif. & Sig. \\
\hline (Constant) & 11.65 & & .000 & 16.59 & & .000 & 4.445 & & .050 \\
$\mathrm{SA}+$ & -6.440 & 6.4400 & .006 & -2.69 & 2.6933 & .463 & 1.540 & -1.5400 & .602 \\
$\mathrm{Cd}_{1}+$ & -10.99 & 10.990 & .000 & -5.77 & 5.7733 & .130 & -.070 & .07000 & .981 \\
$\mathrm{Cd}_{2}+$ & -10.04 & 10.046 & .000 & -8.99 & 8.9933 & .026 & 5.425 & -5.4233 & .084 \\
$\mathrm{Cd}_{1}+\mathrm{SA}$ & -8.470 & 8.4700 & .001 & -4.93 & 4.9333 & .191 & -1.26 & 1.26000 & .669 \\
$\mathrm{Cd}_{2}+\mathrm{SA}$ & -2.975 & 2.9766 & .147 & -4.41 & 4.4100 & .239 & 3.185 & -3.1833 & .290 \\
\hline $\mathrm{R}^{2}$ & \multicolumn{3}{c}{.805} & \multicolumn{3}{c|}{$.375(\mathrm{p}>.05)$} & & & $.381(\mathrm{p}>.05)$ \\
\hline
\end{tabular}

Table 11. Mn contents in leaves of pistachio species under different treatments

\begin{tabular}{|c|c|c|c|c|c|c|c|c|c|}
\hline \multirow{2}{*}{ Mn } & \multicolumn{3}{|c|}{ P. terebinthus } & \multicolumn{3}{|c|}{ P. khinjuk } & \multicolumn{3}{|c|}{ P. vera } \\
\hline & B & Mean Dif. & Sig. & B & Mean Dif. & Sig. & B & Mean Dif. & Sig. \\
\hline (Constant) & 1.610 & & .005 & 4.760 & & .000 & 1.470 & & .223 \\
\hline $\mathrm{SA}+$ & -.140 & .14000 & .837 & -.665 & .66500 & .323 & 13.09 & -13.090 & .000 \\
\hline $\mathrm{Cd}_{1}+$ & 2.870 & -2.8700 & .001 & 1.715 & -1.7150 & .021 & 1.855 & -1.8550 & .273 \\
\hline $\mathrm{Cd}_{2}+$ & 3.290 & -3.2900 & .000 & -2.03 & 2.0300 & .008 & 1.680 & -1.6800 & .319 \\
\hline $\mathrm{Cd}_{1}+\mathrm{SA}$ & 2.240 & -2.2400 & .006 & .945 & -.94500 & .169 & 2.380 & -2.3800 & .167 \\
\hline $\mathrm{Cd}_{2}+\mathrm{SA}$ & .840 & -.8400 & .230 & -.595 & .59500 & .374 & 6.545 & -6.5450 & .002 \\
\hline $\mathrm{R}^{2}$ & \multicolumn{3}{|c|}{.806} & \multicolumn{3}{|c|}{.777} & \multicolumn{3}{|c|}{.882} \\
\hline
\end{tabular}

\section{Cadmium (Cd) content}

$\mathrm{Cd}$ content in leaves significantly decreased with SA alone $(\mathrm{p}=0.001), \mathrm{Cd}_{1}+$ $(\mathrm{p}=0.002)$ whereas the content was not significantly influenced with $\mathrm{Cd}_{2}+(\mathrm{p}=1.000)$, $\mathrm{Cd}_{1}+\mathrm{SA}(\mathrm{p}=0.072)$ and $\mathrm{Cd}_{2}+\mathrm{SA}(\mathrm{p}=0.446)$ for P. terebinthus. For P. khinjuk, $\mathrm{SA}+$ $(\mathrm{p}=0.002), \mathrm{Cd}_{1}+(\mathrm{p}=0.141), \mathrm{Cd}_{2}+(\mathrm{p}=0.001)$ and $\mathrm{Cd} 2+\mathrm{SA}(\mathrm{p}=0.002)$ induced an increase in $\mathrm{Cd}$ content in leaves. However, $\mathrm{Cd} 1+\mathrm{SA}$ did not significantly affect the content $(\mathrm{p}=1.000)$. Interestingly, all treatments quantitatively but non-significantly influenced the Cd content in leaves of P. vera (Table 12). The root samples of maize (Zea mays) treated with SA contained less $\mathrm{Cd}$ than in the plants treated with $\mathrm{Cd}$ alone. However, the highest $\mathrm{Cd}$ content in the leaves was found in SA+Cd plants (Gondor et al., 2016) but $\mathrm{SA}+\mathrm{Cd}_{1}$ or $\mathrm{SA}+\mathrm{Cd}_{2}$ did not exhibit any significant effects regarding $\mathrm{Cd}$ content in leaves of three Pistacia species herein.

\section{Protein content}

Protein content decreased with $\mathrm{SA}+(\mathrm{p}=0.451) \mathrm{Cd}_{1}+(\mathrm{p}=0.140)$ and $\mathrm{Cd}_{2}+(\mathrm{p}=0.000)$ but the content in plants under $\mathrm{Cd}_{1}+$ and $\mathrm{Cd}_{2}+$ treatments coupled with foliar applications of SA increased for $P$. terebinthus. All treatments reduced the protein 
content in P. khinjuk. SA+ increased content when applied with $\mathrm{Cd}_{1}+$ in comparison to the single treatment of $\mathrm{Cd}_{1}+\left(\mathrm{Cd}_{1}+\mathrm{SA} ; \mathrm{p}=0.000\right)$ but $\mathrm{SA}+$ did not cause quantitatively changes when applied with $\mathrm{Cd}_{2}+$ in comparison to the single treatment of $\mathrm{Cd}_{2}+$ $\left(\mathrm{Cd}_{2}+\mathrm{SA} ; \mathrm{p}=0.000\right)$ in P. khinjuk. On the other hand, all treatments except $\mathrm{Cd}_{2}+$ decreased the protein content in $P$. vera. $\mathrm{SA}+$ did not cause quantitatively changes when applied with $\mathrm{Cd}_{1}+$ in comparison to the single treatment of $\mathrm{Cd}_{1}+$ but brought about significant changes when applied with $\mathrm{Cd}_{2}+$ in comparison to the single treatment of $\mathrm{Cd}_{2}+\mathrm{Cd}_{2}+$ significantly increased the content but the interaction of $\mathrm{Cd}_{2}+\mathrm{SA}$ decreased the content $(\mathrm{p}=0.965)$ (Table 13).

Salarizadeh et al. (2016) reporting that the excessive copper decreased the protein content in the pistachio plant but the adverse effects were improved with the addition of SA. The decrease in protein content has been attributed to the toxic effects of heavy metals and their reaction with the $\mathrm{SH}$-groups which subsequently causes protein denaturation (Fuhrer, 1982). Furthermore, the protein content along with the decline in nitrogen content was observed in leaves of wheat seedlings treated with salicylic acid (Singh and Usha, 2003).

Table 12. Cd contents in leaves of pistachio species under different treatments

\begin{tabular}{c|ccc|ccc|ccc}
\hline \multirow{2}{*}{ Cd } & \multicolumn{3}{|c|}{ P. terebinthus } & \multicolumn{3}{c|}{ P. khinjuk } & \multicolumn{3}{c}{ P. vera } \\
\cline { 2 - 10 } & B & Mean Dif. & Sig. & B & Mean Dif. & Sig. & B & Mean Dif. & Sig. \\
\hline (Constant) & .410 & & .000 & .300 & & .000 & .420 & & .000 \\
$\mathrm{SA}+$ & -.110 & .1100 & .001 & .100 & -.100 & .002 & -.020 & .0200 & .446 \\
$\mathrm{Cd}_{1}+$ & -.100 & .1000 & .002 & .040 & -.040 & .141 & -.010 & .0100 & .701 \\
$\mathrm{Cd}_{2}+$ & .000 & .0000 & 1.00 & .110 & -.110 & .001 & -.020 & .0200 & .446 \\
$\mathrm{Cd}_{1}+\mathrm{SA}$ & .050 & -.0500 & .072 & $-1.035 \mathrm{E}-16$ & .000 & 1.00 & -.040 & .0400 & .141 \\
$\mathrm{Cd}_{2}+\mathrm{SA}$ & -.020 & .0200 & .446 & .100 & -.100 & .002 & $-8.614 \mathrm{E}-2$ & 0.000 & 1.00 \\
\hline $\mathrm{R}^{2}$ & \multicolumn{3}{|c|}{.835} & \multicolumn{3}{c|}{.775} & & \multicolumn{3}{c}{$.229(\mathrm{p}>.05)$} \\
\hline
\end{tabular}

Table 13. Protein contents in leaves of pistachio species under different treatments

\begin{tabular}{|c|c|c|c|c|c|c|c|c|c|}
\hline \multirow{2}{*}{ Protein } & \multicolumn{3}{|c|}{ P. terebinthus } & \multicolumn{3}{|c|}{ P. khinjuk } & \multicolumn{3}{|c|}{ P. vera } \\
\hline & B & Mean Dif. & Sig. & B & Mean Dif. & Sig. & B & Mean Dif. & Sig. \\
\hline (Constant) & 10.95 & & .000 & 12.84 & & .000 & 12.18 & & .000 \\
\hline $\mathrm{SA}+$ & -.175 & .18000 & .451 & -3.81 & 3.8100 & .000 & -2.10 & 2.1000 & .000 \\
\hline $\mathrm{Cd}_{1}+$ & -.355 & .35667 & .140 & -2.76 & 2.7633 & .000 & -.180 & .17667 & .438 \\
\hline $\mathrm{Cd}_{2}+$ & -1.580 & 1.5833 & .000 & -1.36 & 1.3633 & .000 & 5.420 & -5.4233 & .000 \\
\hline $\mathrm{Cd}_{1}+\mathrm{SA}$ & .165 & -.16000 & .477 & -1.89 & 1.8900 & .000 & -.185 & .18000 & .426 \\
\hline $\mathrm{Cd}_{2}+\mathrm{SA}$ & 1.040 & -1.0400 & .001 & -1.37 & 1.3700 & .000 & -.010 & .01000 & .965 \\
\hline $\mathrm{R}^{2}$ & & .923 & & & .966 & & & .991 & \\
\hline
\end{tabular}

\section{Correlations}

Herewith correlation analysis, it was aimed to determine whether the coefficient and directions of correlation vary with the pistachio species or not. Hence, correlation analyses for pistachio species were separately performed. Accordingly, correlation coefficients between elements of pistachio species leaves were given in Tables 14-16. According to the correlation matrix of the elements in leaves of $P$. terebinthus, there were negative correlations between $\mathrm{N}$ and $\mathrm{Mn}(\mathrm{r}=-0.606), \mathrm{N}$ and $\mathrm{Cu}(\mathrm{r}=-0.489), \mathrm{N}$ and $\mathrm{Cd}(\mathrm{r}=-0.124), \mathrm{P}$ and $\mathrm{K}(\mathrm{r}=-0.345), \mathrm{P}$ and $\mathrm{Mn}(\mathrm{r}=-0.345), \mathrm{P}$ and $\mathrm{Fe}(\mathrm{r}=-0.042), \mathrm{P}$ and $\mathrm{Fe}(\mathrm{r}=-0.042), \mathrm{P}$ and $\mathrm{Cd}(\mathrm{r}=-0.519), \mathrm{K}$ and $\mathrm{Ca}(\mathrm{r}=-0.796), \mathrm{K}$ and $\mathrm{Mg}(\mathrm{r}=-0.517), \mathrm{K}$ 
and $\mathrm{Zn}(\mathrm{r}=-0.313), \mathrm{Ca}$ and $\mathrm{Mn}(\mathrm{r}=-0.931), \mathrm{Ca}$ and $\mathrm{Cu}(\mathrm{r}=-0.459), \mathrm{Ca}$ and $\mathrm{Fe}(\mathrm{r}=-.316)$, $\mathrm{Ca}$ and $\mathrm{Cd}(\mathrm{r}=-0.966), \mathrm{Mg}$ and $\mathrm{Mn}(\mathrm{r}=-0.657), \mathrm{Mg}$ and $\mathrm{Fe}(\mathrm{r}=-0.656), \mathrm{Mg}$ and $\mathrm{Cd}(\mathrm{r}=$ -0.441), $\mathrm{Zn}$ and $\mathrm{Mn}(\mathrm{r}=-0.845), \mathrm{Zn}$ and $\mathrm{Cu}(\mathrm{r}=-0.816), \mathrm{Zn}$ and $\mathrm{Fe}(\mathrm{r}=-0.001), \mathrm{Zn}$ and $\mathrm{Cd}(\mathrm{r}=-0.612), \mathrm{Cu}$ and $\mathrm{Fe}(\mathrm{r}=-0.393)$. The remained correlation coefficients were positive for P. terebinthus (Table 14).

Table 14. Correlation for P. terebinthus

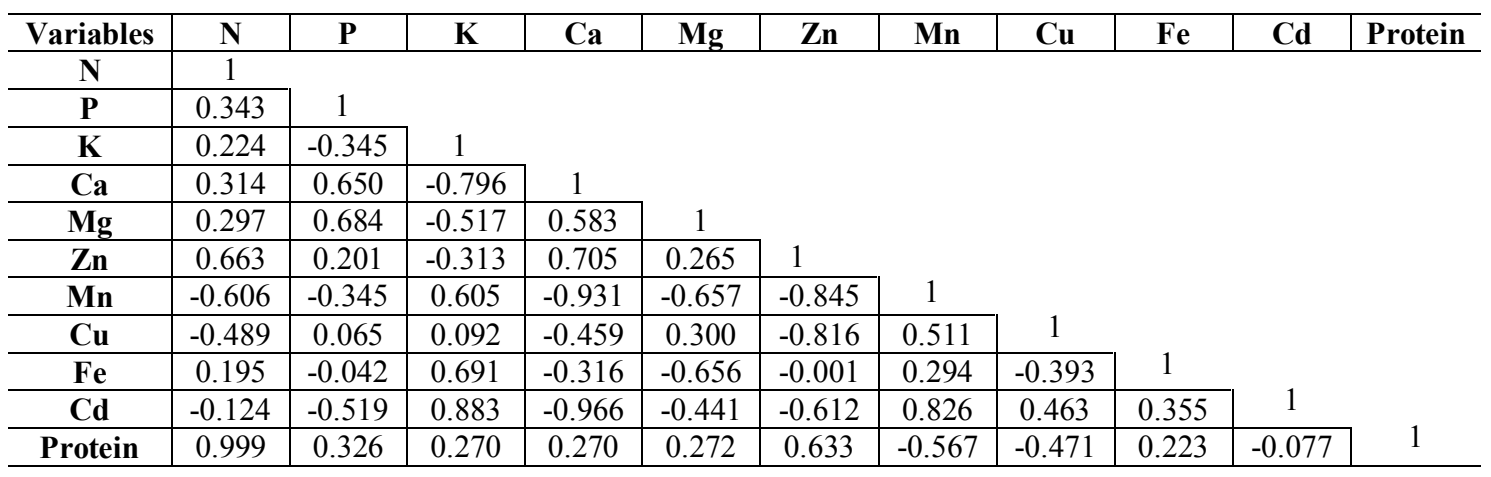

According to the correlation matrix of the elements in leaves of $P$. khinjuk, there were negative correlations between $\mathrm{N}$ and $\mathrm{P}(\mathrm{r}=-0.009), \mathrm{N}$ and $\mathrm{Mn}(\mathrm{r}=-0.186), \mathrm{N}$ and $\mathrm{Cu}(\mathrm{r}=-0.288), \mathrm{N}$ and $\mathrm{Fe}(\mathrm{r}=-0.108), \mathrm{N}$ and $\mathrm{Cd}(\mathrm{r}=-0.380), \mathrm{P}$ and $\mathrm{Mg}(\mathrm{r}=-0.120), \mathrm{P}$ and $\mathrm{Zn}(\mathrm{r}=-0.266), \mathrm{P}$ and $\mathrm{Fe}(\mathrm{r}=-0.733), \mathrm{K}$ and $\mathrm{Fe}(\mathrm{r}=-0.766), \mathrm{Ca}$ and $\mathrm{Fe}(\mathrm{r}=-0.502), \mathrm{Ca}$ and $\mathrm{Cd}(\mathrm{r}=-0.044), \mathrm{Mg}$ and $\mathrm{Mn}(\mathrm{r}=-0.561), \mathrm{Mg}$ and $\mathrm{Fe}(\mathrm{r}=-0.505), \mathrm{Zn}$ and $\mathrm{Cd}(\mathrm{r}=$ -0.472), $\mathrm{Mn}$ and $\mathrm{Cd}(\mathrm{r}=-0.717), \mathrm{Cu}$ and $\mathrm{Fe}(\mathrm{r}=-0.426), \mathrm{Fe}$ and $\mathrm{Cd}(\mathrm{r}=-0.520)$. The remained correlation coefficients were positive for P. khinjuk (Table 15).

Table 15. Correlations for P. khinjuk

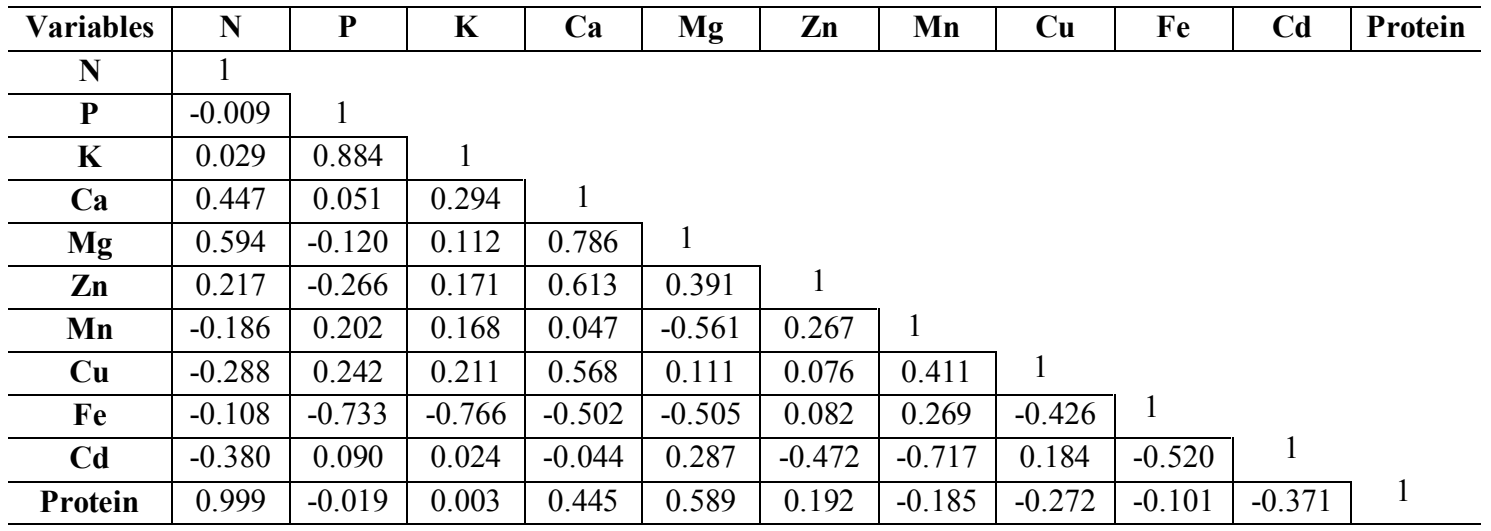

According to the correlation matrix of the elements in leaves of $P$. vera, there were negative correlations between $\mathrm{N}$ and $\mathrm{P}(\mathrm{r}=-0.254), \mathrm{N}$ and $\mathrm{Ca}(\mathrm{r}=-0.466), \mathrm{N}$ and $\mathrm{Mg}(\mathrm{r}=$ $-0.611), \mathrm{N}$ and $\mathrm{Mn}(\mathrm{r}=-0.513), \mathrm{N}$ and $\mathrm{Fe}(\mathrm{r}=-0.202), \mathrm{N}$ and $\mathrm{Cd}(\mathrm{r}=-0.068), \mathrm{P}$ and $\mathrm{Ca}$ $(\mathrm{r}=-0.026), \mathrm{P}$ and $\mathrm{Cu}(\mathrm{r}=-0.755), \mathrm{K}$ and $\mathrm{Ca}(\mathrm{r}=-0.193), \mathrm{K}$ and $\mathrm{Mg}(\mathrm{r}=-0.229), \mathrm{K}$ and $\mathrm{Mn}(\mathrm{r}=-0.253), \mathrm{K}$ and $\mathrm{Fe}(\mathrm{r}=-0.076), \mathrm{K}$ and $\mathrm{Cd}(\mathrm{r}=-0.554), \mathrm{Ca}$ and $\mathrm{Cd}(\mathrm{r}=-0.040), \mathrm{Mg}$ and $\mathrm{Cu}(\mathrm{r}=-0.358), \mathrm{Zn}$ and $\mathrm{Fe}(\mathrm{r}=-0.038), \mathrm{Mn}$ and $\mathrm{Fe}(\mathrm{r}=-0.090), \mathrm{Cu}$ and $\mathrm{Fe}(\mathrm{r}=$ 
-0.677), $\mathrm{Cu}$ and $\mathrm{Cd}(\mathrm{r}=-0.244)$. The remained correlation coefficients were positive for P. vera (Table 16).

Considering all correlation coefficients, Cd negatively correlated with $\mathrm{N}, \mathrm{Ca}$ and protein content in leaves for all three-pistachio species. We should note that those correlations might differ than that of the other tissues of the plants as a consequence of allocation, transport or sequestration of the elements in order to cope with the exogenous treatments.

Table 16. Correlations for P. vera

\begin{tabular}{|c|c|c|c|c|c|c|c|c|c|c|c|}
\hline Variables & $\mathbf{N}$ & $\mathbf{P}$ & K & $\mathrm{Ca}$ & Mg & $\mathbf{Z n}$ & Mn & $\mathbf{C u}$ & $\mathrm{Fe}$ & Cd & Protein \\
\hline $\mathbf{N}$ & 1 & & & & & & & & & & \\
\hline $\mathbf{P}$ & -0.254 & 1 & & & & & & & & & \\
\hline $\mathbf{K}$ & 0.679 & 0.032 & 1 & & & & & & & & \\
\hline $\mathrm{Ca}$ & -0.466 & -0.026 & -0.193 & 1 & & & & & & & \\
\hline Mg & -0.611 & 0.534 & -0.229 & 0.819 & 1 & & & & & & \\
\hline $\mathrm{Zn}$ & 0.699 & 0.186 & 0.423 & 0.049 & 0.080 & 1 & & & & & \\
\hline Mn & -0.513 & 0.321 & -0.253 & 0.809 & 0.909 & 0.158 & 1 & & & & \\
\hline $\mathrm{Cu}$ & 0.441 & -0.755 & 0.000 & 0.036 & -0.358 & 0.305 & 0.020 & 1 & & & \\
\hline $\mathrm{Fe}$ & -0.202 & 0.315 & -0.076 & 0.267 & 0.304 & -0.038 & -0.090 & -0.677 & 1 & & \\
\hline Cd & -0.068 & 0.295 & -0.554 & -0.040 & 0.104 & 0.235 & -0.070 & -0.244 & 0.566 & 1 & \\
\hline Protein & 1.000 & -0.236 & 0.687 & -0.472 & -0.607 & 0.700 & -0.515 & 0.424 & -0.195 & -0.068 & 1 \\
\hline
\end{tabular}

\section{Principal component analysis (PCA)}

The discrimination can be evaluated from the principal component analysis scores plot between pistachio species using identified macro and micro elements as shown in Figs. 1-4. This pair of graphs is a biplot, i.e., macro and micro elements were more expressed in pistachio species leaf samples in the same area of the graph. The experimental groups in each group represent a similar response regarding with element contents, discriminating the experimental group behaviors in response to the treatments. Along with the present study, we discriminated the groups using the macro and micro elements measured. We herein performed four principal component analysis to visualize and discriminate each experimental group (PCA-1: P. terebinthus; PCA-2: P. khinjuk; PCA-3: P. vera; PCA-4: P. terebinthus, P. khinjuk and P. vera). In this context, it was aimed to determine whether the element changes were species or treatment dependent. For $P$. terebinthus $\left(\mathrm{T}-\mathrm{C}, \mathrm{T}+\mathrm{SA}, \mathrm{T}+\mathrm{Cd}_{1}, \mathrm{~T}+\mathrm{Cd}_{2}, \mathrm{~T}+\mathrm{Cd}_{1}+\mathrm{SA}, \mathrm{T}+\mathrm{Cd}_{2}+\mathrm{SA}\right)$, the two principal components accounted for $76.66 \%$ of total variance, whereas the first axis and second axis explained $49.396 \%$ and $26.70 \%$ of total variance (Fig. 1). Experimental groups were well-defined and discriminated, suggesting that addition of the SA did not affect the element content of $P$. terebinthus against $\mathrm{Cd}_{1}$ treatment but significant changes were recorded with the addition of SA to $P$. terebinthus the grown under $\mathrm{Cd}_{2}$ conditions.

For P. khinjuk $\left(\mathrm{K}-\mathrm{C}, \mathrm{K}+\mathrm{SA}, \mathrm{K}+\mathrm{Cd}_{1}, \mathrm{~K}+\mathrm{Cd}_{2}, \mathrm{~K}+\mathrm{Cd}_{1}+\mathrm{SA}, \mathrm{K}+\mathrm{Cd}_{2}+\mathrm{SA}\right)$, the two principal components accounted for $58.18 \%$ of total variance, whereas the first axis and second axis explained $31.98 \%$ and $26.19 \%$ of total variance (Fig. 2). Experimental groups were well-defined and discriminated from control group $(\mathrm{K}-\mathrm{C})$, proposing the responsive structure of $P$. khinjuk against any external stimuli. With addition of SA, the adverse effects of $\mathrm{Cd}_{2}$ were alleviated. 
For P. vera $\left(\mathrm{V}-\mathrm{C}, \mathrm{V}+\mathrm{SA}, \mathrm{V}+\mathrm{Cd}_{1}, \mathrm{~V}+\mathrm{Cd}_{2}, \mathrm{~V}+\mathrm{Cd}_{1}+\mathrm{SA}, \mathrm{V}+\mathrm{Cd}_{2}+\mathrm{SA}\right)$, the two principal components accounted for $60.91 \%$ of total variance, whereas the first axis and second axis explained $40.91 \%$ and $20.00 \%$ of total variance (Fig. 3). Similar responses were recorded in the circumstances of $P$. khinjuk grown under $\mathrm{Cd}_{2}$ and addition of SA.

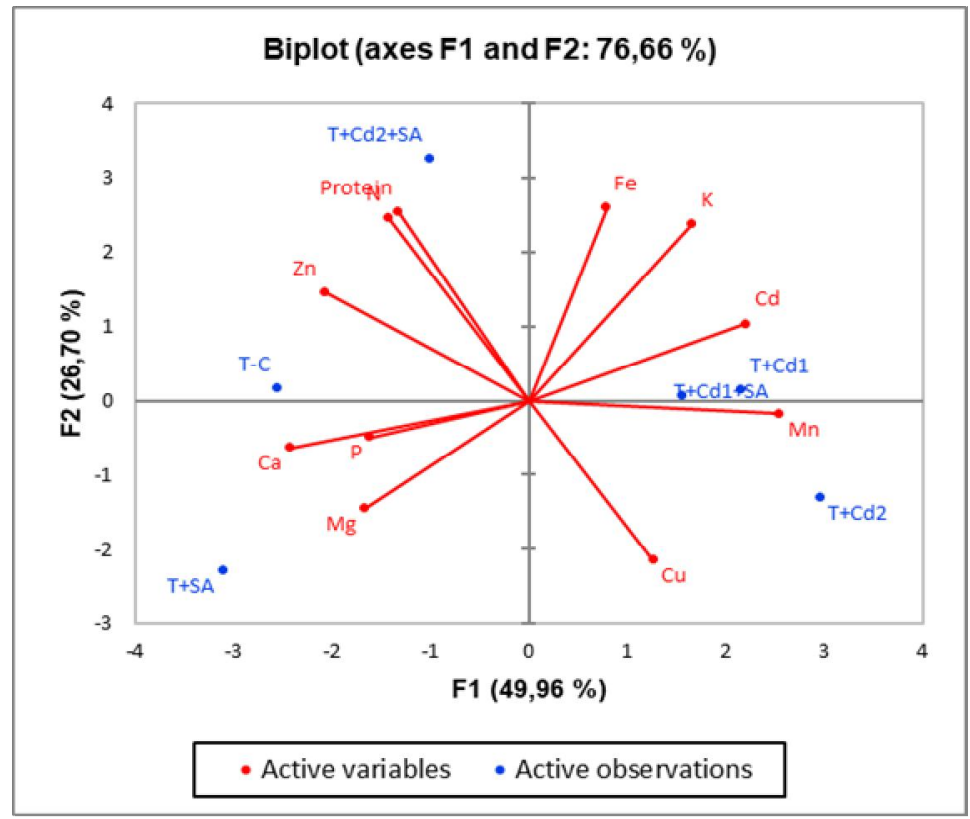

Figure 1. Principal component analysis for P. terebinthus

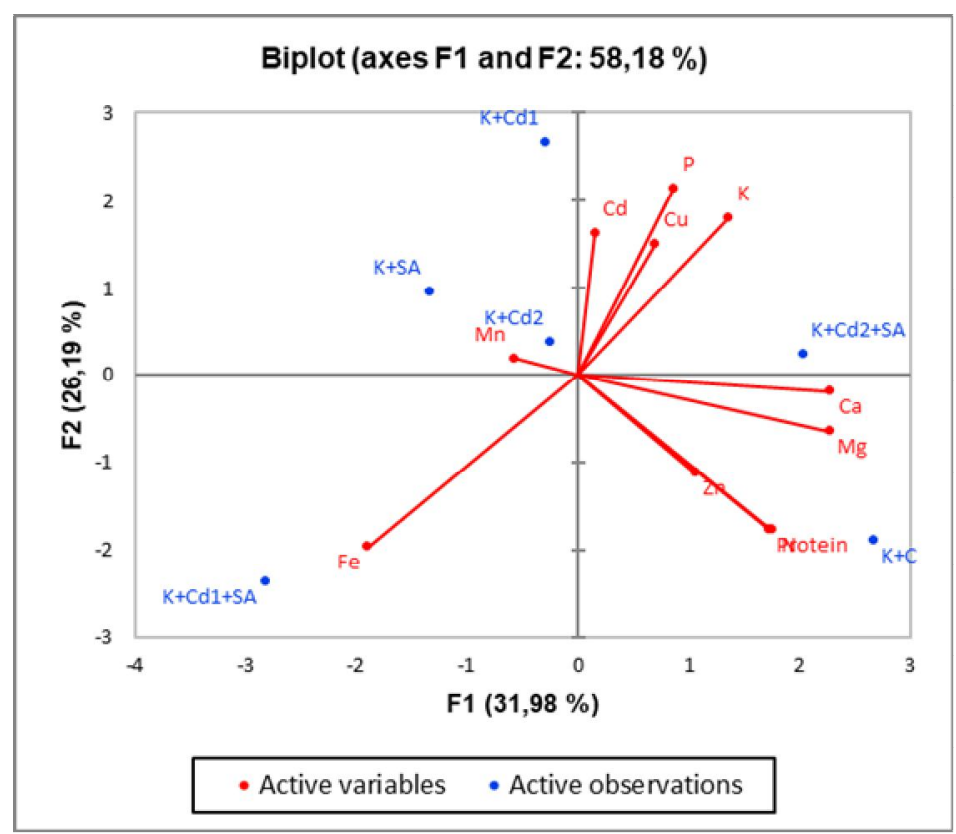

Figure 2. Principal component analysis for P. khinjuk

Considered all experimental groups, results obtained from the principal component analysis showed the presence of the well-discriminated and defined groups for pistachio 
species and exogenous treatments, proposing all species exhibited different mechanisms in accumulation, transport or sequestration of the elements. Along with the visualization provided by principal component analysis, $\mathrm{K}, \mathrm{Zn}$ and $\mathrm{Fe}$ for $P$. khinjuk, $\mathrm{Cu}, \mathrm{Cd}, \mathrm{N}, \mathrm{Mn}$, and $\mathrm{Mg}$ for $P$. vera, $\mathrm{Mn}, \mathrm{Ca}$ and $\mathrm{P}$ for P. terebinthus were more pronounced (Fig. 4).

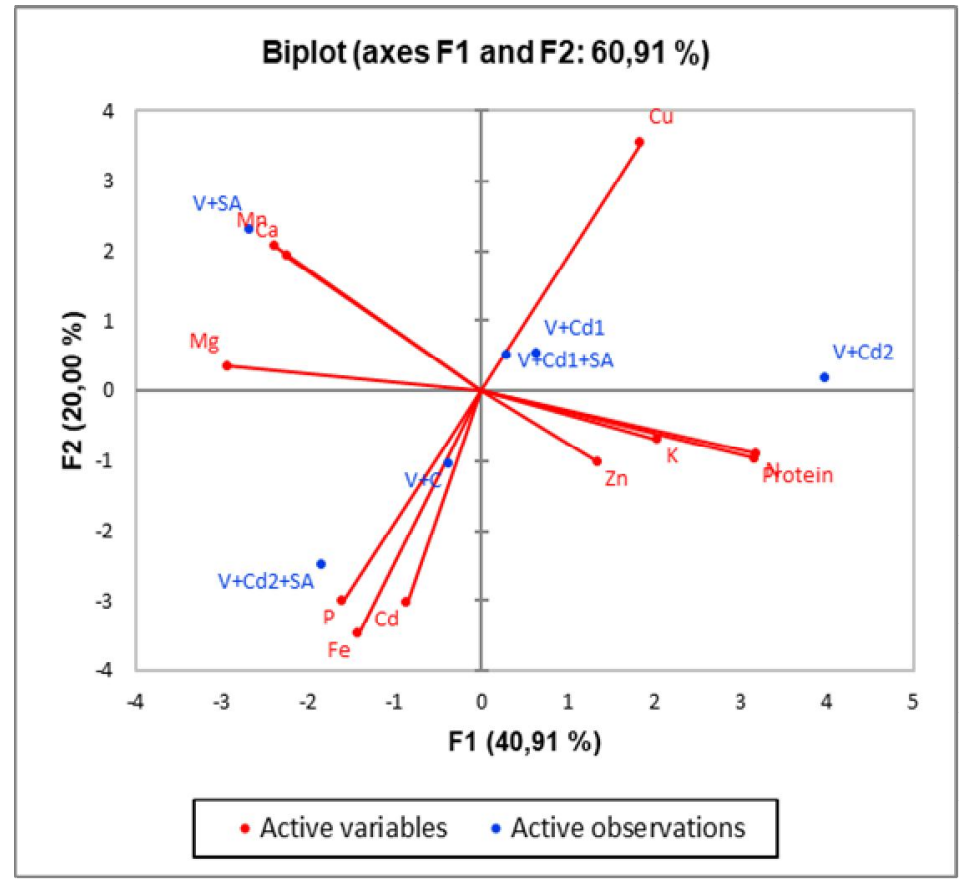

Figure 3. Principal component analysis for P. vera

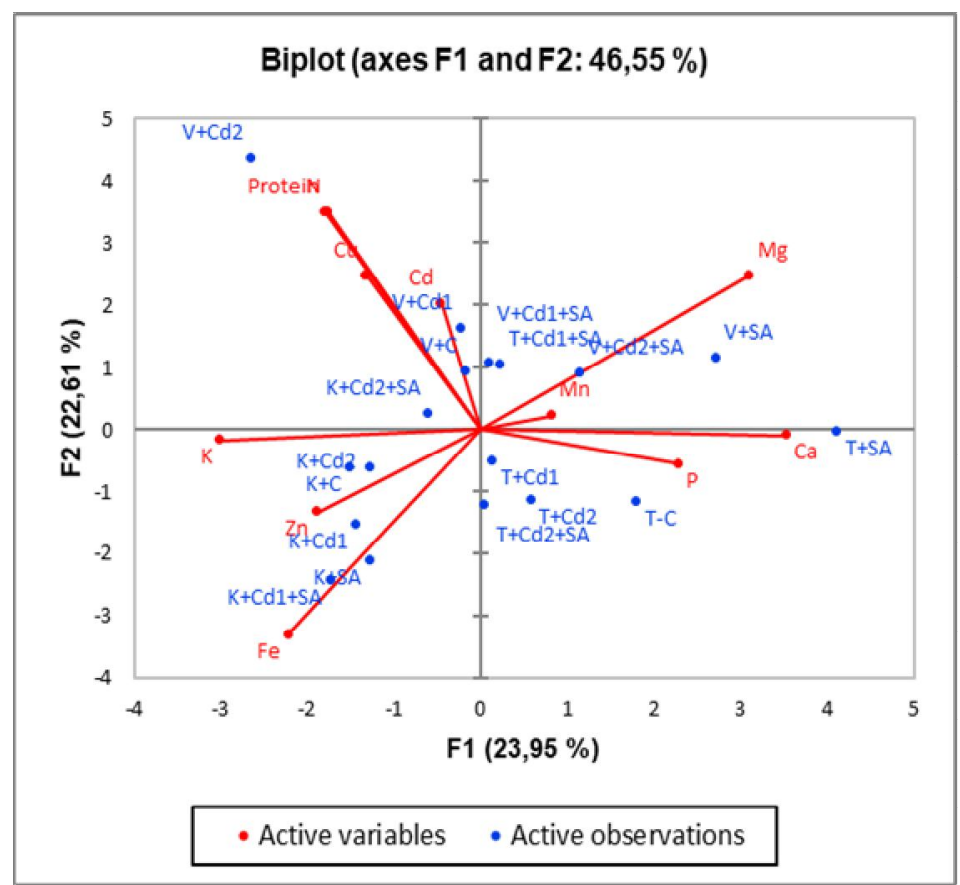

Figure 4. Principal component analysis for three pistachio species exposed to the different treatments 


\section{Prominent and over accumulated elements}

Of the examined elements, $\mathrm{P}$ and $\mathrm{Cu}$ concentrations increased 2.151 and 4.702 times by applications of the cadmium and salicylic acid to pistachio species (Table 17, Figs. 5-6).

Table 17. Over-accumulation of $P$ and $C u$ under various treatments in different pistachio species

\begin{tabular}{c|c|c|c|c|c|c}
\hline Element & Mean (2) & Mean (1) & Log-ratio & Fold change & p & FDR \\
\hline $\mathbf{P}$ & 0.6478 & 0.315 & 0.3328 & 2.151 & 0.0001 & 0.001 \\
\hline $\mathbf{C u}$ & -0.0225 & -0.6723 & 0.6723 & 4.702 & 0.0003 & 0.0017 \\
\hline
\end{tabular}

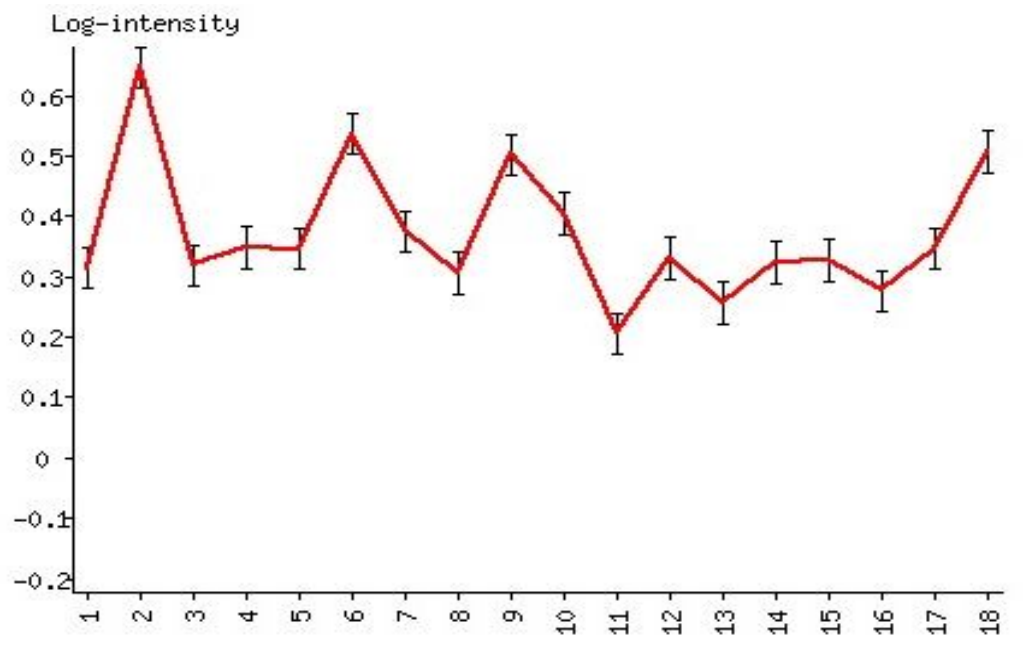

Figure 5. Mean log-intensity for $P$ under various treatments in different pistachio species 1: $T-C ; 2: T+S A ; 3: T+C d_{1} ; 4: T+C d_{2} ; 5: T+C d_{1}+S A ; 6: T+C d_{2}+S A ; 7: K+C ; 8: K+S A$; $9: K+C d 1 ; 10$ : $K+C d_{2} ; 11: K+C d_{1}+S A ; 12: K+C d_{2}+S A ; 13: V+C ; 14: V+S A ; 15: V+C d_{1} ; 16: V+C d_{2} ; 17: V+C d_{1}+S A$; 18: $V+C d_{2}+S A$

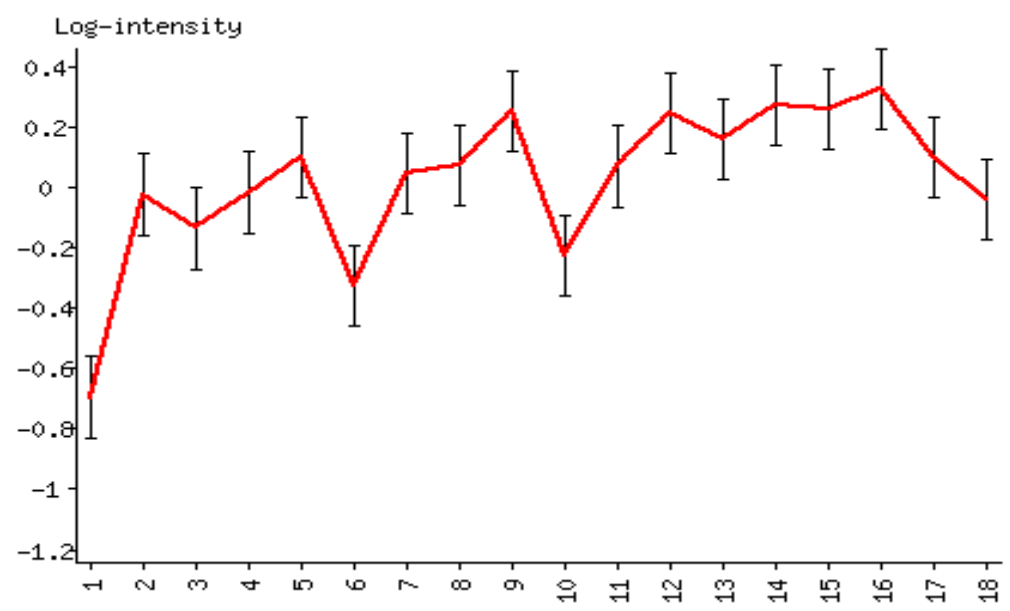

Figure 6. Mean log-intensity for $C u$ under various treatments in different pistachio species 1: $T-C$; 2: $T+S A ; 3: T+C d_{1} ; 4: T+C d_{2} ; 5: T+C d_{1}+S A ; 6: T+C d_{2}+S A ; 7: K+C ; 8: K+S A$; 9: $K+C d 1 ; 10$ : $K+C d_{2} ; 11: K+C d_{1}+S A ; 12: K+C d_{2}+S A ; 13: V+C ; 14: V+S A ; 15: V+C d_{1} ; 16: V+C d_{2} ; 17: V+C d_{1}+S A$; 18: $\mathrm{V}+\mathrm{Cd}+\mathrm{SA}$ 
Phosphorus $(\mathrm{P})$, as phosphate $\left(\mathrm{PO}_{4}^{-3}\right)$, is a complementary component of important compounds that form plant cells, including sugar-phosphates and phospholipids that form the plant membrane as intermediates in respiration and photosynthesis. Since it is an important part of DNA and RNA molecules, it has been reported that phosphorspecific genes are necessary in the expression mechanism and may be involved in the regulation of enzyme reactions as part of proteins (Dordas, 2009). In addition, phosphorus, as an element of ATP, helps transform energy in many biochemical events (Sieprawska et al., 2014). In the case of arid regions or any disruption in the roots of the plant, the phosphate content decreases in the tissues of the plant. Due to this decrease, the rate of photosynthesis per unit in leaves is also significantly affected. It was reported that the decrease in leaf growth and photosynthetic ratio due to phosphorus may be related to permeability of stomata and ribulose 1,5 biphosphate carboxylase regeneration capacity (Brooks, 1986).

Of the microelements, copper $(\mathrm{Cu})$ plays significant roles in regulation of photosynthesis, respiration, antioxidant activity, cell wall metabolism and hormone perception. Furthermore, $\mathrm{Cu}$ is a cofactor of various enzymes such as copper/zinc superoxide dismutase ( $\mathrm{Cu} / \mathrm{ZnSOD})$, cytochrome-c oxidase (Pilon et al., 2006).

\section{Discussion}

Exogenous SA applications have been determined to increase the development and photosynthetic efficiency in plants such as Oryza sativa (Chen et al., 2007), Zea mays (Krantev et al., 2008), Phasealus vulgaris (Zengin, 2014) which have been exposed to various heavy metals. It has also been shown to be effective on antioxidant mechanism by lowering membrane lipid peroxidation (Chen et al., 2007; Zengin, 2014). Moreover, SA application regulates various metabolic processes in plants, encourages the production of osmolyte and secondary metabolites, adjusts the nutrient status and protects the plant under abiotic stress conditions (Khan et al., 2015). SA triggered high production coupled with high concentration of photosynthetic pigments, photosynthetic activities and higher antioxidant enzymes in plants exposed to Cd stress (Zhang et al., 2015). As a regulatory role of SA, Studies have shown that SA applications are an important elicitor that regulates photosynthesis, photosystem II, photosynthetic pigments and enzyme activities in plants under metal stress and controls the formation of $\mathrm{H}_{2} \mathrm{O}_{2}$ and gives endurance by controlling the formation of $\mathrm{H}_{2} \mathrm{O}_{2}$ (Al-Whaibi et al., 2012; Noriega et al., 2012; Belkadhi et al., 2014; Zhang et al., 2015).

Furthermore, SA has a role in alleviating heavy metal toxicity (Shi and Zhu, 2008; Zhou et al., 2009; Wang et al., 2013). In addition, it has been determined that SA acts as a regulator in the increase of antioxidant enzymes and in the absorption and distribution of nutrients by reducing the Mn transport from the roots to the shoot (Sheng et al., 2015).

Of the studies carried out on the accumulation of nutrients, the content of elements exhibited different responses. Application of SA in salt stressed plants caused a decrease in $\mathrm{K}, \mathrm{Ca}$ and $\mathrm{P}$ content (El-Tayeb, 2005). However, exogenous SA treatments in wheat caused increases in $\mathrm{P}, \mathrm{K}, \mathrm{Ca}$ and $\mathrm{Mg}$ contents (Aldesuquy et al., 2012; Hassanein et al., 2012; Loutfy et al., 2012).

Along with the current study, the effect of salicylic acid treatments on the element content of pistachio species was different. According to the results, SA application increased $\mathrm{P}, \mathrm{Ca}, \mathrm{Mg}$ and $\mathrm{Cu}$ content and decreased $\mathrm{N}$ content in Pistacia terebinthus. 
While Fe content increased in Pistacia khinjuk, the other elements decreased. In Pistacia vera, $\mathrm{P}, \mathrm{Ca}, \mathrm{Mg}, \mathrm{Zn}, \mathrm{Mn}, \mathrm{Cu}$ content increased.

$50 \mu \mathrm{M}$ cadmium $\left(\mathrm{Cd}_{1}\right)$ reduced the content of most elements. As a result of $\mathrm{Cd}_{1}$ treatment, Pistacia terebinthus has increased $\mathrm{K}, \mathrm{Mn}, \mathrm{Cu}, \mathrm{Fe}$ and $\mathrm{Cd}$ uptake while other elements and protein have decreased or remained at the same level. While $\mathrm{P}, \mathrm{Mn}, \mathrm{Cu}$ and $\mathrm{Cd}$ accumulation increased, $\mathrm{N}, \mathrm{Ca}, \mathrm{Mg}, \mathrm{Zn}, \mathrm{Fe}$ and protein decreased in Pistacia khinjuk. $\mathrm{P}, \mathrm{Mn}$, and $\mathrm{Cu}$ content increased while $\mathrm{N}, \mathrm{K}, \mathrm{Ca}, \mathrm{Mg}$ and $\mathrm{Fe}$ content decreased in Pistacia vera.

Three pistachio species exhibited different responses against $100 \mu \mathrm{M}$ cadmium $\left(\mathrm{Cd}_{2}\right)$ treatment. As a result of treatment, $\mathrm{K}, \mathrm{Mn}, \mathrm{Cu}, \mathrm{Fe}$ and $\mathrm{Cd}$ content increased while $\mathrm{N}$, $\mathrm{Ca}, \mathrm{Mg}, \mathrm{Zn}$, and protein decreased in Pistacia terebinthus. $\mathrm{P}$ content exhibited an increase whereas other element and protein content decreased in Pistacia khinjuk. In Pistacia vera, $\mathrm{N}, \mathrm{K}, \mathrm{Zn}, \mathrm{Mn}, \mathrm{Cu}$ and protein content increased while $\mathrm{Ca}, \mathrm{Mg}$ and $\mathrm{Fe}$ content decreased.

Pistachio species exhibited different responses concerned with the concentration of element under $50 \mu \mathrm{M}$ with addition of SA $\left(\mathrm{Cd}_{1}+\mathrm{SA}\right)$. As a result of the treatment, the concentration of $\mathrm{K}, \mathrm{Mg}, \mathrm{Mn}, \mathrm{Cu}$, protein and $\mathrm{Cd}$ increased in P. terebinthus. In P. khinjuk, $\mathrm{Mn}$ and $\mathrm{Fe}$ concentration increased whereas other elements concentration and protein content decreased. Furthermore, $\mathrm{K}, \mathrm{Mg}, \mathrm{Mn}$ and protein content increased while $\mathrm{N}, \mathrm{Ca}, \mathrm{Zn}, \mathrm{Cu}, \mathrm{Fe}$ and $\mathrm{Cd}$ concentration decreased in $P$. vera.

Herewith the correlation analysis, pistachio species exhibited different behaviors regarding with correlations for their element contents, which might be deemed as consequences of uptake and accumulation in response to the exogenous treatments.

\section{Conclusion}

To sum up, pistachio species exhibited different responses with respect to the content of elements and proteins in their leaf tissues under cadmium and salicylic acid treatments. Also, species responded differently against cadmium concentrations and salicylic acid. Generally, the changes in content of elements were more pronounced by $100 \mu \mathrm{M} \mathrm{Cd}$ treatment. Although the response of the species was different, addition of SA positively affected the element and protein content. While cadmium and salicylic acid affected the content of elements in the leaf, the effect of salicylic acid on cadmium was not significant.

Differences concerned with element content and responses against foliar SA among cultivars were revealed and discriminated via chemometric techniques. Also, possible active roles of $\mathrm{P}$ and $\mathrm{Cu}$ against $\mathrm{Cd}$ and $\mathrm{SA}$ applications were also determined for pistachio species. By applications of the cadmium and salicylic acid to pistachio species, leaves' $\mathrm{P}$ content 2.151 times and leaves' $\mathrm{Cu}$ content 4.702 times were increased, suggesting the forthcoming studies to be concentrated on the exogenous applications of $\mathrm{P}$ or $\mathrm{Cu}$ in order to improve the responses of pistachio species against $\mathrm{Cd}$ stress.

Furthermore, these results might be considered to be significant in terms of the selection and use of rootstocks of pistachio species by considering the different reactions regarding with the element and protein content. Subsequently, the elementmediated or induced growth parameters might also be considered under unfavorable environmental conditions. 
Acknowledgements. This study was supported by Scientific Research Foundation Unit of Kilis 7 Aralik University with the code 2013/01/MAP/03.

\section{REFERENCES}

[1] Acar, I., Kafkas, S., Kapchina-Toteva, V., Ercisli, S. (2017a): Effect of rootstock on fat content and fatty acid composition of immature pistachio kernels. - Comptes rendus de l'Académie Bulgare des Sciences 70(7): 1049-1056.

[2] Acar, I., Yasar, H., Ercisli, S. (2017b): Effects of dormancy-breaking treatments on seed germination and seedling growth of Pistacia khinjuk Stocks using as rootstock for pistachio trees. - Journal of Applied Botany and Food Quality 90: 191-196.

[3] Aldesuquy, H. S., Abbas, M. A., Abo-Hamed, S. A., Elhakem, A. H., Alsokari, S. S. (2012): Glycine betaine and salicylic acid induced modification in productivity of two different cultivars of wheat grown under water stress. - Journal of Stress Physiology and Biochemistry 8(2): 72-89.

[4] Al-Whaibi, M. H., Siddiqui, M. H., Basalah, M. O. (2012): Salicylic acid and calciuminduced protection of wheat against salinity. - Protoplasma 249: 769-778.

[5] Belkadhi, A., DeHaro, A., Obregon, S., Chaïbi, W., Djebali, W. (2014): Positive effects of salicylic acid pretreatment on the composition of flaxplastidial membrane lipids under cadmium stress. - Environmental Science and Pollution Research 22: 1457-1467.

[6] Bhupinder, S., Usha, K. (2003): Salicylic acid induced physiological and biochemical changes in wheat seedlings under water stress. - Plant Growth Regulation 39: 137-41.

[7] Brooks, A. (1986): Effects of phosphorus nutrition on ribulose-1, 5-bisphosphate carboxylase activation, photosynthetic quantum yield and amounts of some Calvin-cycle metabolites in spinach leaves. - Functional Plant Biology 13(2): 221-237.

[8] Chapman, H. D., Pratt, P. F. (1961): Methods of Analysis for Soils, Plants and Waters. University of California, Division of Agricultural Sciences, USA.

[9] Chen, J., Zhu, C., Li, L., Sun, Z., Pan, X. (2007): Effects of exogenous salicylic acid on growth and $\mathrm{H}_{2} \mathrm{O}_{2}$-metabolizing enzymes in rice seedlings under lead stress. - Journal of Environmental Science 19: 44-49.

[10] di Toppi, L. S., Gabbrielli, R. (1999): Response to Cadmium in Higher Plants. Environmental and Experimental Botany 41(2): 105-130.

[11] Dordas, C. (2009): Dry matter, nitrogen and phosphorus accumulation, partitioning and remobilization as affected by $\mathrm{N}$ and $\mathrm{P}$ fertilization and source-sink relations. - European Journal of Agronomy 30(2): 129-139.

[12] El-Tayeb, M. A. (2005): Response of barley grains to the interactive effect of salinity and salicylic acid. - Plant Growth Regulation 45(3): 215-224.

[13] El-Tayeb, M. A., Ahmed, N. L. (2010): Response of wheat cultivars to drought and salicylic acid. - American-Eurasian Journal of Agronomy 3(1): 1-7.

[14] Esmail-Pour, A. (2001): Distribution, use and conservation of pistachio in Iran. In Toward a Comprehensive Documentation and Use of Pistacia Genetic Diversity in Central and West Asia, North Africa and Europe. - Report of the IPGRI Workshop: 1626.

[15] Feng, J., Shi, Q., Wang, X., Wei, M., Yang, F., Xu, H. (2010): Silicon Supplementation Ameliorated the Inhibition of Photosynthesis and Nitrate Metabolism by Cadmium (Cd) Toxicity in Cucumis sativus L. - Scientia Horticulturae 123(4): 521-530.

[16] Fuhrer, J. (1982): Early effects of excess cadmium uptake in Phaseolus vulgaris. - Plant Cell and Environment 5: 263-270.

[17] Ganesan, V., Thomas, G. (2001): Salicylic acid response in rice: influence of salicylic acid on $\mathrm{H}_{2} \mathrm{O}_{2}$ accumulation and oxidative stress. - Plant Science 160(6): 1095-1106. 
[18] Gondor, O. K., Pál, M., Darkó, É., Janda, T., Szalai, G. (2016): Salicylic acid and sodium salicylate alleviate cadmium toxicity to different extents in maize (Zea mays L.). - PloS ONE 11(8): 1-18.

[19] Hassanein, R. A., Abdelkader, A. F., Ali, H., Amin, A. A. E. S., Mohammed Rashad, E. S. (2012): Grain-priming and foliar pretreatment enhanced stress defense in wheat (Triticum aestivum var. Gimaza 9) plants cultivated in drought land. - Australian Journal of Crop Science 6(1): 121-129.

[20] Hossain, M. A., Hasanuzzaman, M., Fujita, M. (2010): Up Regulation of Antioxidant and Glyoxalase Systems by Exogenous Glycinebetaine and Proline in Mung Bean Confer Tolerance to Cadmium Stress. - Physiology and Molecular Biology of Plants 16(3): 259272.

[21] Kacar, B., Inal, A. (2008): Plant Analysis. - Nobel Press, (Bitki Analizleri. Nobel Yayınları) Ankara.

[22] Khan, N. A., Singh, S., Nazar, R. (2007): Activities of Antioxidative Enzymes, Sulphur Assimilation, Photosynthetic Activity and Growth of Wheat (Triticum aestivum) Cultivars Differing in Yield Potential under Cadmium Stress. - Journal of Agronomy and Crop Science 193(6): 435-444.

[23] Khan, M. I. R., Fatma, M., Per, T. S., Anjum, N. A., Khan, N. A. (2015): Salicylic acidinduced abiotic stress tolerance and underlying mechanisms in plants. - Frontiers in Plant Science 6: 1-17.

[24] Klessig, D. F., Malamy, J. (1994): The salicylic acid signal in plants. - Plant Molecular Biology 26(5): 1439-1458.

[25] Krantev, A., Yordanova, R., Janda, T., Szalai, G., Popova, L. (2008): Treatment with salicylic acid decreases the effect of cadmium on photosynthesis in maize plants. Journal of Plant Physiology 165: 920-931.

[26] Kulak, M. (2018): A bibliometric review of research trends in salicylic acid uses in agricultural and biological sciences: where have been studies directed? - Scientific Papers. Series A. Agronomy LXI(1): 296-303.

[27] Loutfy, N., El-Tayeb, M. A., Hassanen, A. M., Moustafa, M. F., Sakuma, Y., Inouhe, M. (2012): Changes in the water status and osmotic solute contents in response to drought and salicylic acid treatments in four different cultivars of wheat (Triticum aestivum). Journal of Plant Research 125(1): 173-184.

[28] McGrath, S. P., Zhao, F. J., Lombi, E. (2001): Plant and rhizosphere processes involved in phytoremediation of metal-contaminated soils. - Plant and Soil 232(1-2): 207-214.

[29] Mishra, A., Choudhuri, M. A. (1999): Effects of salicylic acid on heavy metal-induced membrane deterioration mediated by lipoxygenase in rice. - Biologia Plantarum 42(3): 409-415.

[30] Mobin, M., Khan, N. A. (2007): Photosynthetic Activity, Pigment Composition and Antioxidative Response of Two Mustard (Brassica juncea) Cultivars Differing in Photosynthetic Capacity Subjected to Cadmium Stress. - Journal of Plant Physiology 164(5): 601-610.

[31] Noriega, G., Caggiano, E., Lecube, M. L., Santa Cruz, D., Batlle, A., Tomaro, M., Balestrasse, K. B. (2012): The role of salicylic acid in the prevention of oxidative stress elicited by cadmium in soybean plants. - Biometals 25(6): 1155-1165.

[32] Pál, M. (2002): Effect of salicylic acid during heavy metal stress. - Acta Biologica Szegediensis 46(3-4): 119-120.

[33] Pilon, M., Abdel-Ghany, S. E., Cohu, C. M., Gogolin, K. A., Ye, H. (2006): Copper cofactor delivery in plant cells. - Current Opinion in Plant Biology 9(3): 256-263.

[34] Raskin, I. (1992): Role of salicylic acid in plants. - Annual Review of Plant Biology 43(1): 439-463.

[35] Sakhabutdinova, A. R., Fatkhutdinova, D. R., Bezrukova, M. V., Shakirova, F. M. (2003): Salicylic acid prevents the damaging action of stress factors on wheat plants. Bulgarian Journal of Plant Physiology 21: 314-319. 
[36] Salarizadeh, M. R., Saeidisar, S., Abbaspour, H., Hokmabadi, H. (2016): The Effects of Spermine and Salicylic Acid on Pistachio (Pistacia vera L.) Cultivars (Badami and Qazvini) under Copper Stress. - Journal of Nuts 7(2): 89-99.

[37] Savvas, D., Colla, G., Rouphael, Y., Schwarz, D. (2010): Amelioration of heavy metal and nutrient stress in fruit vegetables by grafting. - Scientia Horticulturae 127: 156-161.

[38] Savvas, D., Ntatsi, G., Moiras, N., Tsakalidis, A., Ropokis, A., Liopa-Tsakalidi, A. (2011): Impact of grafting and rootstock on the responses of cucumber to heavy metal stress. - In V Balkan Symposium on Vegetables and Potatoes 960: 49-56.

[39] Shakirova, F. M., Bezrukova, M. V. (1997): Induction of wheat resistance against environmental salinization by indolylacetic acid. - Izvestiya Akademii Nauk Seriya Biologicheskaya 2: 149-153.

[40] Shakirova, F. M., Sakhabutdinova, A. R., Bezrukova, M. V., Fatkhutdinova, R. A., Fatkhutdinova, D. R. (2003): Changes in the hormonal status of wheat seedlings induced by salicylic acid and salinity. - Plant Science 164(3): 317-322.

[41] Sheng, H., Zeng, J., Yan, F., Wang, X., Wang, Y., Kang, H., Fan, X., Sha, L., Zhang, H., Zhou, Y. (2015): Effect of exogenous salicylic acid on manganese toxicity, mineral nutrients translocation and antioxidative system in polish wheat (Triticum polonicum L.). - Acta Physiologiae Plantarum 37(2): 1-11.

[42] Shi, Q., Zhu, Z. (2008): Effects of exogenous salicylic acid on manganese toxicity, element contents and antioxidative system in cucumber. - Environmental and Experimental Botany 63: 317-326.

[43] Shi, G., Liu, C., Cai, Q., Liu, Q., Hou, C. (2010): Cadmium accumulation and tolerance of two safflower cultivars in relation to photosynthesis and antioxidative enzymes. Bulletin of Environmental Contamination and Toxicology 85(3): 256-263.

[44] Sieprawska, A., Filek, M., Walas, S., Tobiasz, A., Mrowiec, H., Miszalski, Z. (2014): Does micro-and macroelement content differentiate grains of sensitive and tolerant wheat varieties? - Acta Physiologiae Plantarum 36(11): 3095-3100.

[45] Singh, B., Usha, K. (2003): Salicylic acid induced physiological and biochemical changes in wheat seedlings under water stress. - Plant Growth Regulation 39(2): 137-141.

[46] Wang, C., Zhang, S., Wang, P., Hou, J., Qian, J., Ao, Y., Li, L. (2011): Salicylic acid involved in the regulation of nutrient elements uptake and oxidative stress in Vallisneria natans (Lour.) Hara under Pb stress. - Chemosphere 84(1): 136-142.

[47] Wang, Q., Liang, X., Dong, Y., Xu, L., Zhang, X., Kong, J., Liu, S. (2013): Effects of exogenous salicylic acid and nitric oxide on physiological characteristics of perennial ryegrass under cadmium stress. - Journal of Plant Growth Regulators 32: 721-731.

[48] Webster, A. D. (1995): Rootstock and interstock effects on deciduous fruit tree vigour, precocity, and yield productivity. - New Zealand Journal of Crop and Horticultural Science 23: 373-382.

[49] Zengin, F. (2014): Exogenous treatment with salicylic acid alleviating copper toxicity in bean seedlings. - Proceedings of the National Academy of Sciences, India Section B: Biological Sciences 84(3): 749-755.

[50] Zhang, Y., Xu, S., Yang, S., Chen, Y. (2015): Salicylic acid alleviates cadmium-induced inhibition of growth and photosynthesis through up regulating antioxidant defense system in two melon cultivars (Cucumis melo L.). - Protoplasma 252(3): 911-924.

[51] Zhou, Z. S., Guo, K., Elbaz, A. A., Yang, Z. M. (2009): Salicylic acid alleviates mercury toxicity by preventing oxidative stress in roots of Medicago sativa. - Environmental and Experimental Botany 65: 27-34. 